\title{
STATIC BENDING OF ISOTROPIC CIRCULAR CYLINDRICAL SHELLS BASED ON THE HIGHER ORDER SHEAR DEFORMATION THEORY OF REDDY AND LIU
}

\author{
C.U. NWOJI \\ Department of Civil Engineering \\ University of Nigeria, Nsukka, Enugu state, NIGERIA \\ D.G. ANI \\ Department of Civil Engineering \\ University of Nigeria, Nsukka, Enugu state, NIGERIA \\ O.A. OGUAGHAMBA \\ Department of Civil Engineering \\ University of Nigeria, Nsukka, Enugu state, NIGERIA \\ V.T. IBEABUCHI* \\ Department of Civil Engineering \\ Alex Ekwueme Federal University Ndufu Alike \\ P.M.B. 1010, Abakaliki, NIGERIA \\ E-mail: ibeabuchi.victor@funai.edu.ng
}

\begin{abstract}
In this paper, a displacement based shear deformation theory formulated on the cubic in-plane displacement field equation of Reddy and Liu is presented for the static bending analysis of isotropic circular cylindrical shells. The adopted displacement field accounts for a quadratic (parabolic) distribution of the transverse shear through the shell thickness as well as satisfies the need for a stress free upper and lower boundary surfaces of the shell. The equations of static equilibrium are obtained on application of the principle of virtual work. Numerical results of the bending analysis for the displacements and stresses are presented for the simply supported shell. A comparison made to those of the Kirchhoff-Love theory for varying shell length to mean - radius of curvature ratios, shows good agreement for thin shells irrespective of the shell length to radius of curvature ratio $(l / a)$. The transverse sharing effect is found to be noticeable in the deformation of thick shells, however, this effect diminishes with a continuous increase in $l / a$ ratios.
\end{abstract}

Key words: isotropic circular cylindrical shell, shear deformation theory, transverse shear, principle of virtual work, Kirchhoff- Love theory.

\section{Introduction}

Shell structures are three-dimensional solids closely bounded by two arbitrary curved surfaces whose thickness is comparatively small in relation to its other dimensions. A good number of shell theories were proposed with the aim of predicting more adequately the elastic behavior of a shell in response to both static and dynamic loadings, with significant progress recorded thus far. Structural shells are generally classified in terms of their thickness to radius of curvature ratios $\left(h / R_{i}\right)$ as thin and thick shells, with thin shells defined

\footnotetext{
${ }^{*}$ To whom correspondence should be addressed
} 
in ratios $h / R_{i} \leq 1 / 20$ as in Novozhilov [1]. The classical shell theories (CSTs) otherwise known as the first approximation shell theories are founded on the kinematic postulation of Kirchhoff-Love, where the shell is assumed to be thin. The Kirchhoff-Love postulation is based on the following:

(1) straight lines lying normal to the middle surface of the shell prior to deformation remain unchanged after the deformation has occurred.

(2) the stresses generated normal to the shell's middle surface are considered negligible.

(3) the shell is thin, with the generated displacements and strains considered small.

Shell theories developed upon the Kirchhoff-Love assumptions have been found to provide satisfactory results for shells classified as thin, irrespective of the fundamental flaw associated with the neglect of the transverse shear effects on the shell's deformation as proposed by the first assumption stated above. However, applications of these fundamental assumptions to anisotropic composites or thick shells have resulted in erroneous results in displacements, frequencies of vibrations, buckling loads and stresses. With this in mind other categories of structural shell theories have been developed to arrest these shortcomings. The first order shear deformation theories (FSDTs) founded on Mindlin's postulation attempted to address this issue, by accounting for shear deformation through the relaxing of the assumption associated with the preservation of the normals to the middle surface. Mindlin [2] achieved this by further suggesting that these normals indeed remain straight, however are not necessarily normal to the deformed middle surface. These shell theories based on Mindlin hypothesis have been found to result in quite satisfactory outcomes regarding the prediction of the state of stress within thin and moderately thick shells. However, they have been shown to be inadequate for proper estimation of the transverse shear distribution. A limitation which arose was the need for transverse shear correction coefficients to account for its erring constant distribution of the transverse shear as opposed to the parabolic distribution predicted by the theory of elasticity. Due to this unfortunate drawback associated with the FSDTs an additional category of shell theories emerged known as the higher order shear deformation theories (HSDTs). These shell theories are not restricted to using only linear terms, but second and higher order terms are expanded as functions of the shell's thickness coordinate, with the out-of-plane displacement (deflection) assumed to be constant through the shell's thickness. This assumption of constant deflection is equivalent to the neglect of the stretching of the normals ensuring that the shell deformation problem remains a two dimensional one [3, 4].

Reddy [5] applied the FSDT to analyze the deflection and vibrational frequencies of doubly curved laminated shells using two sets of transverse shear correction coefficients to examine their influence on the fundamental frequencies. Quite remarkable discoveries were documented with the selection of these shear correction factors proven to grossly affect the accuracy of the FSDT. Soldatos [6] employed the FSDT to the free vibrational analysis of isotropic cylindrical shells of oval cross section with the value of the shear correction coefficient proposed by Mindlin. The HSDTs have been able to overcome the challenge associated with the use of shear correction factors by adopting displacement fields of cubic and higher order functions, which accounts for a more accurate quadratic distribution of the transverse shear with the stress free surface boundary condition also met. These higher order theories involve those proposed in Reddy and Liu [7] for studying the shear deformation of laminated shells of orthotropic layers as applied in $[6,8,9]$ to study the shear deformation of noncircular cylindrical, laminated anisotropic and symmetric laminated shells. Recent work by Vuong and Duc [10], applied these theories the analytical investigation on buckling and post-buckling of shear deformable sandwich toroidal shell segments with functionally graded core and homogeneous face sheets.

The present study however investigated the transverse shearing effects on the static bending behavior of isotropic circular cylindrical shells based on the third order displacement field of Reddy and Liu. Hence, the present solution is unaffected by the challenges associated with the use of transverse shear correction factors.

\section{Theoretical formulation}

The present work is an extension of the shear deformation theory developed by Reddy and Liu [7] for doubly curved laminated composites to the static bending problem of an elastic isotropic circular cylindrical shell. The solution is formulated upon a cubic function expansion of the in-plane displacement field together with a constant through thickness deflection. The fundamental assumptions of the present work would mostly 
retain the classical theory assumptions with its only deviation being the adoption of a parabolic distribution of the transverse shearing stresses, together with the satisfaction of the zero stress surface conditions. The shell thickness is to be considered small enough in comparison to the radii of curvature (i.e $h / R_{i}<<1$ ) with the material considered homogenous, isotropic and obeying Hooke's law. The equation of kinematics (strain field) is to be obtained upon substitution of the displacement field into the known strain - displacement relations for a shell in an orthogonal curvilinear coordinate system. The stress field is obtained upon consideration of the strain field and the known material stress - strain laws. The governing differential equations of equilibrium associated with the adopted displacement field would then be obtained using the principle of virtual work.

Let $\left(\alpha_{1}, \alpha_{2}, \alpha_{3}\right)$ be introduced as an orthogonal curvilinear coordinate system as depicted in Fig.1., such that $\left(\alpha_{1}, \alpha_{2}\right)$ are parametric lines of the curvature lying in the middle surface $\alpha_{3} \approx 0$ of the shell. The infinitesimal distance between two points on the shell's middle surface as in Ventsel and Krauthammer [11] is given as:

$$
(d s)^{2}=A^{2} d \alpha_{1}^{2}+B^{2} d \alpha_{2}^{2}
$$

where Eq.(2.1) is known as the fundamental form and the quantities $A$ and $B$ are the fundamental form parameters or Lame's parameters.

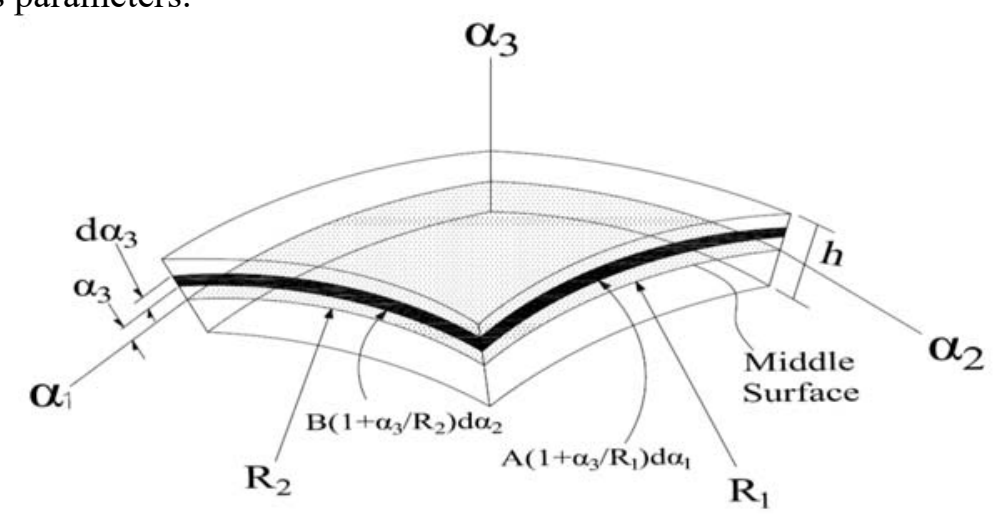

Fig.1. Differential element of a shell.

\subsection{Kinematics}

The assumed displacement field is in the form as given by Reddy and Liu [7]

$$
\begin{aligned}
& \bar{U}\left(\alpha_{1}, \alpha_{2}, \alpha_{3}\right)=\left(1+\frac{\alpha_{3}}{R_{1}}\right) u+\alpha_{3} \varphi_{1}-\frac{4 \alpha_{3}^{3}}{3 h^{2}}\left(\varphi_{1}+\frac{1}{A} \frac{\partial w}{\partial \alpha_{1}}\right), \\
& \bar{V}\left(\alpha_{1}, \alpha_{2}, \alpha_{3}\right)=\left(1+\frac{\alpha_{3}}{R_{2}}\right) v+\alpha_{3} \varphi_{2}-\frac{4 \alpha_{3}^{3}}{3 h^{2}}\left(\varphi_{2}+\frac{1}{B} \frac{\partial w}{\partial \alpha_{2}}\right), \\
& \bar{W}\left(\alpha_{1}, \alpha_{2}, 0\right)=w
\end{aligned}
$$

where $(\bar{U}, \bar{V}, \bar{W})$ are the components of the displacements in the orthogonal $\left(\alpha_{1}, \alpha_{2}, \alpha_{3}\right)$ coordinates, $(u, v, w)$ are the displacements at a point $\left(\alpha_{1}, \alpha_{2}, 0\right)$ on the shell middle surface, $\left(\varphi_{1}, \varphi_{2}\right)$ are the rotations of normals at $\alpha_{3}=0$ corresponding to the $\alpha_{2}$ and $\alpha_{1}$ axis while $\alpha_{3}$ is the thickness coordinate. 
In Eqs (2.2) and (2.3) the cubic form of the in- plane displacements provides a parabolic (quadratic) distribution of the transverse shear through the shells thickness and is found to result in zero transverse shear strains at the upper and lower surfaces of the shell $\left(1\right.$.e $\left.\alpha_{3}= \pm h / 2\right)$, hence satisfying the stress free surface requirement.

The known strain - displacement relations for a shell in the orthogonal curvilinear coordinate system as in Viola et al. [12] with the modifications to the transverse shear strains by Reddy and Liu [7] incorporated, are given as follows:

$$
\begin{aligned}
\varepsilon_{11} & =\frac{1}{1+\alpha_{3} / R_{1}}\left(\frac{1}{A} \frac{\partial \bar{U}}{\partial \alpha_{1}}+\frac{\bar{V}}{A B} \frac{\partial A}{\partial \alpha_{2}}+\frac{\bar{W}}{R_{1}}\right), \\
\varepsilon_{22} & =\frac{1}{1+\alpha_{3} / R_{2}}\left(\frac{1}{B} \frac{\partial \bar{V}}{\partial \alpha_{2}}+\frac{\bar{U}}{A B} \frac{\partial B}{\partial \alpha_{1}}+\frac{\bar{W}}{R_{2}}\right), \\
\varepsilon_{12} & =\frac{1}{1+\alpha_{3} / R_{1}}\left(\frac{1}{A} \frac{\partial \bar{V}}{\partial \alpha_{1}}-\frac{\bar{U}}{A B} \frac{\partial A}{\partial \alpha_{2}}\right)+\frac{1}{1+\alpha_{3} / R_{2}}\left(\frac{1}{B} \frac{\partial \bar{U}}{\partial \alpha_{2}}-\frac{\bar{V}}{A B} \frac{\partial B}{\partial \alpha_{1}}\right), \\
\varepsilon_{13} & =\frac{1}{1+\alpha_{3} / R_{1}}\left(\frac{1}{A} \frac{\partial \bar{W}}{\partial \alpha_{1}}-\frac{u}{R_{1}}\right)+\frac{\partial \bar{U}}{\partial \alpha_{3}}, \\
\varepsilon_{23} & =\frac{1}{1+\alpha_{3} / R_{2}}\left(\frac{1}{B} \frac{\partial \bar{W}}{\partial \alpha_{2}}-\frac{v}{R_{2}}\right)+\frac{\partial \bar{V}}{\partial \alpha_{3}}, \\
\varepsilon_{33} & =\frac{\partial \bar{W}}{\partial \alpha_{3}} .
\end{aligned}
$$

The assumption associated with the shell thickness $h / R_{i}<<1$ makes it necessary to drop the following terms $\alpha_{3} / R_{i}<<1$. This assumption also makes possible the neglect of the normal strain effect $\left(\varepsilon_{33}=0\right)$ together with the normal stress effect $\left(\sigma_{33}=0\right)$. According to Amabili and Reddy [4], the normal stress has been verfied to be small when compared to the transverse shear stresses, except near the edges of the shell. Hence the plane stress assumption $\left(\sigma_{33}=0\right)$ is a good approximation of the stress state in a moderately thick shell, to which the following thickness to radius of curvature ratios are valid $\frac{1}{100} \leq \frac{h}{R_{i}} \leq \frac{1}{10}$.

The strain displacements relations for the shell problem are obtained upon substitution of the displacement field Eqs (2.2)-(2.4) into the kinematic relations Eqs (2.5)-(2.9). The strain field is obtained as follows:

$$
\begin{aligned}
& \left\{\begin{array}{l}
\varepsilon_{11} \\
\varepsilon_{22} \\
\varepsilon_{12}
\end{array}\right\}=\left\{\begin{array}{l}
\varepsilon_{11}^{(0)} \\
\varepsilon_{22}^{(0)} \\
\varepsilon_{12}^{(0)}
\end{array}\right\}+\alpha_{3}\left\{\begin{array}{l}
\kappa_{11}^{(0)} \\
\kappa_{22}^{(0)} \\
\kappa_{12}^{(0)}
\end{array}\right\}+\alpha_{3}^{3}\left\{\begin{array}{c}
\kappa_{11}^{(2)} \\
\kappa_{22}^{(2)} \\
\kappa_{12}^{(2)}
\end{array}\right\}, \\
& \left\{\begin{array}{l}
\varepsilon_{13} \\
\varepsilon_{23}
\end{array}\right\}=\left\{\begin{array}{l}
\varepsilon_{13}^{(0)} \\
\varepsilon_{23}^{(0)}
\end{array}\right\}+\alpha_{3}^{2}\left\{\begin{array}{c}
\kappa_{13}^{(I)} \\
\kappa_{23}^{(I)}
\end{array}\right\}
\end{aligned}
$$


with the strain components given below as

$$
\begin{aligned}
& \varepsilon_{11}^{(0)}=\frac{1}{A} \frac{\partial u}{\partial \alpha_{1}}+\frac{v}{A B} \frac{\partial A}{\partial \alpha_{2}}+\frac{w}{R_{1}}, \quad \kappa_{11}^{(0)}=\frac{1}{A} \frac{\partial \varphi_{1}}{\partial \alpha_{1}}+\frac{\varphi_{2}}{A B} \frac{\partial A}{\partial \alpha_{2}}, \\
& \kappa_{11}^{(2)}=-\frac{4}{3 h^{2}}\left[\frac{1}{A} \frac{\partial \varphi_{1}}{\partial \alpha_{1}}+\frac{1}{A B} \frac{\partial A}{\partial \alpha_{2}} \frac{1}{B} \frac{\partial w}{\partial \alpha_{2}}+\frac{\varphi_{2}}{A B} \frac{\partial A}{\partial \alpha_{2}}+\frac{1}{A} \frac{\partial}{\partial \alpha_{1}}\left(\frac{1}{A} \frac{\partial w}{\partial \alpha_{1}}\right)\right] \text {, } \\
& \varepsilon_{22}^{(0)}=\frac{1}{B} \frac{\partial v}{\partial \alpha_{2}}+\frac{u}{A B} \frac{\partial B}{\partial \alpha_{1}}+\frac{w}{R_{2}}, \quad \kappa_{22}{ }^{(0)}=\frac{1}{B} \frac{\partial \varphi_{2}}{\partial \alpha_{2}}+\frac{\varphi_{1}}{A B} \frac{\partial B}{\partial \alpha_{1}} \\
& \kappa_{22}^{(2)}=-\frac{4}{3 h^{2}}\left[\frac{1}{B_{1}} \frac{\partial \varphi_{2}}{\partial \alpha_{2}}+\frac{1}{A B} \frac{\partial B}{\partial \alpha_{1}} \frac{1}{A} \frac{\partial w}{\partial \alpha_{1}}+\frac{\varphi_{1}}{A B} \frac{\partial B}{\partial \alpha_{1}}+\frac{1}{B} \frac{\partial}{\partial \alpha_{2}}\left(\frac{1}{B} \frac{\partial w}{\partial \alpha_{2}}\right)\right], \\
& \varepsilon_{12}^{(0)}=\frac{1}{A} \frac{\partial v}{\partial \alpha_{1}}-\frac{u}{A B} \frac{\partial A}{\partial \alpha_{2}}+\frac{1}{B} \frac{\partial u}{\partial \alpha_{2}}-\frac{v}{A B} \frac{\partial B}{\partial \alpha_{1}} \\
& \kappa_{12}{ }^{(0)}=\frac{1}{A} \frac{\partial \varphi_{2}}{\partial \alpha_{1}}-\frac{\varphi_{1}}{A B} \frac{\partial A}{\partial \alpha_{2}}+\frac{1}{B} \frac{\partial \varphi_{1}}{\partial \alpha_{2}}-\frac{\varphi_{2}}{A B} \frac{\partial B}{\partial \alpha_{1}}, \\
& \kappa_{12}^{(2)}=-\frac{4}{3 h^{2}}\left[\frac{1}{A} \frac{\partial \varphi_{2}}{\partial \alpha_{1}}+\frac{1}{A} \frac{\partial}{\partial \alpha_{1}}\left(\frac{1}{B} \frac{\partial w}{\partial \alpha_{2}}\right)-\frac{\varphi_{1}}{A B} \frac{\partial A}{\partial \alpha_{2}}-\frac{1}{A B} \frac{\partial A}{\partial \alpha_{2}} \frac{1}{A} \frac{\partial w}{\partial \alpha_{1}}+\right. \\
& \left.+\frac{1}{B} \frac{\partial \varphi_{1}}{\partial \alpha_{2}}+\frac{1}{B} \frac{\partial}{\partial \alpha_{2}}\left(\frac{1}{A} \frac{\partial w}{\partial \alpha_{1}}\right)-\frac{\varphi_{2}}{A B} \frac{\partial B}{\partial \alpha_{1}}-\frac{1}{A B} \frac{\partial B}{\partial \alpha_{1}} \frac{1}{B} \frac{\partial w}{\partial \alpha_{2}}\right] \\
& \varepsilon_{13}{ }^{(0)}=\varphi_{1}+\frac{1}{A} \frac{\partial w}{\partial \alpha_{1}}, \quad \kappa_{13}{ }^{(1)}=-\frac{4}{h^{2}}\left(\varphi_{1}+\frac{1}{A} \frac{\partial w}{\partial \alpha_{1}}\right), \quad \varepsilon_{23}{ }^{(0)}=\varphi_{2}+\frac{1}{B} \frac{\partial w}{\partial \alpha_{2}} \\
& \kappa_{23}^{(l)}=-\frac{4}{h^{2}}\left(\varphi_{2}+\frac{1}{B} \frac{\partial w}{\partial \alpha_{2}}\right)
\end{aligned}
$$

\subsection{Constitutive equations, stress field and stress resultants}

The stress - strains laws for an isotropic, homogenous material as in Soedel [13] with the transverse normal stress taken as zero $\left(\sigma_{33}=0\right)$ are given by:

$$
\begin{aligned}
& \sigma_{11}=\frac{E}{1-\mu^{2}}\left(\varepsilon_{11}+\mu \varepsilon_{22}\right), \quad \sigma_{22}=\frac{E}{1-\mu^{2}}\left(\varepsilon_{22}+\mu \varepsilon_{11}\right), \quad \sigma_{12}=\frac{E}{2(1+\mu)} \varepsilon_{12}, \\
& \sigma_{13}=\frac{E}{2(1+\mu)} \varepsilon_{13}, \quad \sigma_{23}=\frac{E}{2(1+\mu)} \varepsilon_{23}
\end{aligned}
$$


where $\mu$ is the poisons ratio, $\frac{E}{2(1+\mu)}$ is the shear modulus and $E$ is the modulus of elasticity.

The stress field is obtained by the substitution of the expressions of the strain field Eqs (2.11) and (2.12) into the constitutive laws Eq.(2.14), the expressions of the stress field may then be obtained as follows:

$$
\begin{aligned}
& \sigma_{11}=\frac{E}{1-\mu^{2}}\left[\varepsilon_{11}^{(0)}+\mu \varepsilon_{22}^{(0)}+\alpha_{3}\left(\kappa_{11}^{(0)}+\mu \kappa_{22}^{(0)}\right)+\alpha_{3}^{3}\left(\kappa_{11}^{(2)}+\mu \kappa_{22}^{(2)}\right)\right] \\
& \sigma_{22}=\frac{E}{1-\mu^{2}}\left[\varepsilon_{22}^{(0)}+\mu \varepsilon_{11}^{(0)}+\alpha_{3}\left(\kappa_{22}^{(0)}+\mu \kappa_{11}^{(0)}\right)+\alpha_{3}^{3}\left(\kappa_{22}^{(2)}+\mu \kappa_{11}^{(2)}\right)\right], \\
& \sigma_{12}=\frac{E}{2(1+\mu)}\left[\varepsilon_{12}^{(0)}+\alpha_{3} \kappa_{12}^{(0)}+\alpha_{3}^{3} \kappa_{12}^{(2)}\right], \\
& \sigma_{13}=\frac{E}{2(1+\mu)}\left[\varepsilon_{13}^{(0)}+\alpha_{3}^{2} \kappa_{13}^{(I)}\right] \\
& \sigma_{23}=\frac{E}{2(1+\mu)}\left[\varepsilon_{23}^{(0)}+\alpha_{3}^{2} \kappa_{23}^{(1)}\right] .
\end{aligned}
$$

The expression of the stress resultants are obtained by the following integrals of the stress fields:

$$
\begin{aligned}
& \left(N_{11}, M_{11}, P_{11}\right)=\int_{-h / 2}^{h / 2} \sigma_{11}\left(1, \alpha_{3}, \alpha_{3}{ }^{3}\right) d \alpha_{3}, \quad\left(N_{12}, M_{12}, P_{12}\right)=\int_{-h / 2}^{h / 2} \sigma_{12}\left(1, \alpha_{3}, \alpha_{3}{ }^{3}\right) d \alpha_{3} \\
& \left(N_{22}, M_{22}, P_{22}\right)=\int_{-h / 2}^{h / 2} \sigma_{22}\left(1, \alpha_{3}, \alpha_{3}^{3}\right) d \alpha_{3}, \quad\left(Q_{13}, R_{13}\right)=\int_{-h / 2}^{h / 2} \sigma_{13}\left(1, \alpha_{3}^{2}\right) d \alpha_{3}, \\
& \left(Q_{23}, R_{23}\right)=\int_{-h / 2}^{h / 2} \sigma_{23}\left(1, \alpha_{3}^{2}\right) d \alpha_{3}
\end{aligned}
$$

where $N, M, P, Q, R$ are the stress resultants.

By substitution of the stress fields, into Eq.(2.20) and performing the integration, the expressions of the stress resultants are then obtained as follows:

$$
\begin{aligned}
& N_{11}=K\left(\varepsilon_{11}{ }^{(0)}+\mu \varepsilon_{22}{ }^{(0)}\right), \\
& N_{22}=K\left(\varepsilon_{22}{ }^{(0)}+\mu \varepsilon_{11}{ }^{(0)}\right), \\
& N_{12}=\frac{K(1-\mu)}{2} \varepsilon_{12}{ }^{(0)}, \\
& M_{11}=D\left(\kappa_{11}{ }^{(0)}+\mu \kappa_{22}{ }^{(0)}\right)+F\left(\kappa_{11}{ }^{(2)}+\mu \kappa_{22}{ }^{(2)}\right), \\
& M_{22}=D\left(\kappa_{22}{ }^{(0)}+\mu \kappa_{11}{ }^{(0)}\right)+F\left(\kappa_{22}{ }^{(2)}+\mu \kappa_{11}{ }^{(2)}\right),
\end{aligned}
$$




$$
\begin{aligned}
& M_{12}=\frac{D(1-\mu)}{2} \kappa_{12}{ }^{(0)}+\frac{F(1-\mu)}{2} \kappa_{12}{ }^{(2)}, \\
& P_{11}=F\left(\kappa_{11}{ }^{(0)}+\mu \kappa_{22}{ }^{(0)}\right)+H\left(\kappa_{11}{ }^{(2)}+\mu \kappa_{22}{ }^{(2)}\right), \\
& P_{22}=F\left(\kappa_{22}{ }^{(0)}+\mu \kappa_{11}{ }^{(0)}\right)+H\left(\kappa_{22}{ }^{(2)}+\mu \kappa_{11}{ }^{(2)}\right), \\
& P_{12}=\frac{F(1-\mu)}{2} \kappa_{12}{ }^{(0)}+\frac{H(1-\mu)}{2} \kappa_{12}^{(2)}, \\
& Q_{13}=\frac{K(1-\mu)}{2} \varepsilon_{13}{ }^{(0)}+\frac{D(1-\mu)}{2} \kappa_{13}{ }^{(I)}, \\
& Q_{23}=\frac{K(1-\mu)}{2} \varepsilon_{23}{ }^{(0)}+\frac{D(1-\mu)}{2} \kappa_{23}^{(l)}, \\
& R_{13}=\frac{D(1-\mu)}{2} \varepsilon_{13}{ }^{(0)}+\frac{F(1-\mu)}{2} \kappa_{13}^{(l)}, \\
& R_{23}=\frac{D(1-\mu)}{2} \varepsilon_{23}{ }^{(0)}+\frac{F(1-\mu)}{2} \kappa_{23}{ }^{(1)}
\end{aligned}
$$

where $K, D, F$ and $H$ are the shell stiffnesses given by

$$
K, D, F, H=\frac{E}{1-\mu^{2}}\left[h, \frac{h^{3}}{12}, \frac{h^{5}}{80}, \frac{h^{7}}{448}\right] .
$$

Solving Eqs (2.21)-(2.33) for the strain components, the stress field Eqs (2.15)-(2.19) may then be obtained in stress resultants as follows:

$$
\begin{aligned}
& \sigma_{11}=\frac{E}{1-\mu^{2}}\left\{\frac{N_{11}}{K}+\alpha_{3}\left(\frac{M_{11}}{D}-\frac{F}{D}\left[\frac{P_{11} D-M_{11} F}{H D-F^{2}}\right]\right)+\alpha_{3}^{3}\left(\frac{P_{11} D-M_{11} F}{H D-F^{2}}\right)\right\}, \\
& \sigma_{22}=\frac{E}{1-\mu^{2}}\left\{\frac{N_{22}}{K}+\alpha_{3}\left(\frac{M_{22}}{D}-\frac{F}{D}\left[\frac{P_{22} D-M_{22} F}{H D-F^{2}}\right]\right)+\alpha_{3}^{3}\left(\frac{P_{22} D-M_{22} F}{H D-F^{2}}\right)\right\}, \\
& \sigma_{12}=\frac{E}{1+\mu}\left\{\frac{N_{12}}{K(1-\mu)}+\alpha_{3}\left[\frac{M_{12}}{D(1-\mu)}-\frac{F}{D}\left(\frac{P_{12} D-M_{12} F}{\left(H D-F^{2}\right)(1-\mu)}\right)\right]+\right. \\
& \left.+\alpha_{3}^{3}\left[\frac{P_{12} D-M_{12} F}{\left(H D-F^{2}\right)(1-\mu)}\right]\right\},
\end{aligned}
$$




$$
\begin{aligned}
& \sigma_{13}=\frac{E}{1+\mu}\left\{\frac{Q_{13}}{K(1-\mu)}-\frac{D}{K}\left(\frac{R_{13} K-Q_{13} D}{\left(F K-D^{2}\right)(1-\mu)}\right)+\alpha_{3}^{2}\left[\frac{R_{13} K-Q_{13} D}{\left(F K-D^{2}\right)(1-\mu)}\right]\right\}, \\
& \sigma_{23}=\frac{E}{1+\mu}\left\{\frac{Q_{23}}{K(1-\mu)}-\frac{D}{K}\left(\frac{R_{23} K-Q_{23} D}{\left(F K-D^{2}\right)(1-\mu)}\right)+\alpha_{3}^{2}\left[\frac{R_{23} K-Q_{23} D}{\left(F K-D^{2}\right)(1-\mu)}\right]\right\},
\end{aligned}
$$

with definitions of the stiffnesses given by Eq.(2.34) substituted into Eqs (2.35)-(2.39), the stress field becomes:

$$
\begin{aligned}
& \sigma_{11}=\frac{N_{11}}{h}+\alpha_{3}\left(\frac{75 M_{11}}{h^{3}}-\frac{420 P_{11}}{h^{5}}\right)+\alpha_{3}^{3}\left(\frac{2800 P_{11}}{h^{7}}-\frac{420 M_{11}}{h^{5}}\right), \\
& \sigma_{22}=\frac{N_{22}}{h}+\alpha_{3}\left(\frac{75 M_{22}}{h^{3}}-\frac{420 P_{22}}{h^{5}}\right)+\alpha_{3}^{3}\left(\frac{2800 P_{22}}{h^{7}}-\frac{420 M_{22}}{h^{5}}\right), \\
& \sigma_{11}=\frac{N_{11}}{h}+\alpha_{3}\left(\frac{75 M_{12}}{h^{3}}-\frac{420 P_{12}}{h^{5}}\right)+\alpha_{3}^{3}\left(\frac{2800 P_{12}}{h^{7}}-\frac{420 M_{12}}{h^{5}}\right), \\
& \sigma_{13}=\frac{9 Q_{13}}{4 h}-\frac{15 R_{13}}{h^{3}}+\alpha_{3}^{2}\left(\frac{180 R_{13}}{h^{5}}-\frac{15 Q_{13}}{h^{3}}\right) \\
& \sigma_{23}=\frac{9 Q_{23}}{4 h}-\frac{15 R_{23}}{h^{3}}+\alpha_{3}^{2}\left(\frac{180 R_{23}}{h^{5}}-\frac{15 Q_{23}}{h^{3}}\right) .
\end{aligned}
$$

\subsection{Equations of static equilibrium}

The principle of virtual work can be applied efficiently in deriving the equations of static problems of continuum as in Ibeabuchi et al. [14] and Ibearugbulem et al. [15]. This principle of virtual work is used in deriving the equations of equilibrium associated with the displacements field in Eqs (2.2)-(2.4). The principle of virtual work stated in an analytic form as in Soedel [13] can be expressed as:

$$
\delta w=\delta w_{I}+\delta w_{E}=0
$$

with the virtual work due to the internal and external forces given respectively as:

$$
\begin{aligned}
& \delta w_{I}=\int_{\alpha_{1}} \int_{\alpha_{2}} \int_{\alpha_{3}}\left(\sigma_{11} \delta \varepsilon_{11}+\sigma_{22} \delta \varepsilon_{22}+\sigma_{12} \delta \varepsilon_{12}+\right. \\
& \left.++\sigma_{13} \delta \varepsilon_{13}+\sigma_{23} \delta \varepsilon_{23}\right) A B d \alpha_{1} d \alpha_{2} d \alpha_{3}, \\
& \delta w_{E}=-\int_{\alpha_{1}} \int_{\alpha_{2}}\left(q_{1} \delta u+q_{2} \delta v+q_{3} \delta w\right) A B d \alpha_{1} d \alpha_{2},
\end{aligned}
$$

with $q_{1}, q_{2}$ and $q_{3}$ being the distributed loads.

The principle of virtual work when applied to the present study results in 


$$
\begin{aligned}
& 0=\int_{\alpha_{1}} \int_{\alpha_{2}}\left[\int_{-h / 2}^{h / 2}\left(\sigma_{11} \delta \varepsilon_{11}+\sigma_{22} \delta \varepsilon_{22}+\sigma_{12} \delta \varepsilon_{12}+\sigma_{13} \delta \varepsilon_{13}+\sigma_{23} \delta \varepsilon_{23}\right) d \alpha_{3}+\right. \\
& \left.-\left(q_{1} \delta u+q_{2} \delta v+q_{3} \delta w\right)\right] A B d \alpha_{1} d \alpha_{2}=\int_{\alpha_{1}} \int_{\alpha_{2}}\left[N_{11} \delta \varepsilon_{11}^{(0)}+M_{11} \delta \kappa_{11}^{(0)}+P_{11} \delta \kappa_{11}^{(2)}+\right. \\
& +N_{22} \delta \varepsilon_{22}^{(0)}+M_{22} \delta \kappa_{22}^{(0)}+P_{22} \delta \kappa_{22}^{(2)}+N_{12} \delta \varepsilon_{12}^{(0)}+M_{12} \delta \kappa_{12}^{(0)}+P_{12} \delta \kappa_{12}^{(2)}+Q_{13} \delta \varepsilon_{13}^{(0)}+ \\
& \left.+R_{13} \delta \kappa_{13}^{(1)}+Q_{23} \delta \varepsilon_{23}^{(0)}+R_{23} \delta \kappa_{23}^{(l)}-q_{1} \delta u-q_{2} \delta v-q_{3} \delta w\right] A B d \alpha_{1} d \alpha_{2} .
\end{aligned}
$$

Substituting the strain field components in Eq.(2.13) into Eq.(2.48) and with the displacement gradients integrated by parts, the governing equations of static equilibrium are obtained from the resulting expression by setting the coefficients of variations $\delta u, \delta v, \delta w, \delta \varphi_{1}$ and $\delta \varphi_{2}$ to zero separately:

$$
\begin{aligned}
& \delta u: \frac{\partial\left(N_{11} B\right)}{\partial \alpha_{1}}-N_{22} \frac{\partial B}{\partial \alpha_{1}}+\frac{\partial\left(N_{21} A\right)}{\partial \alpha_{2}}+N_{12} \frac{\partial A}{\partial \alpha_{2}}+q_{1} A B=0, \\
& \delta v: \frac{\partial\left(N_{22} A\right)}{\partial \alpha_{2}}-N_{11} \frac{\partial A}{\partial \alpha_{2}}+\frac{\partial\left(N_{12} B\right)}{\partial \alpha_{1}}+N_{21} \frac{\partial B}{\partial \alpha_{1}}+q_{2} A B=0, \\
& \delta \mathrm{w}: C_{1} \frac{\partial^{2} P_{11}}{\partial \alpha_{1}^{2}} \frac{B}{A}-N_{11} \frac{A B}{R_{1}}-C_{1} \frac{\partial P_{11}}{\partial \alpha_{2}} \frac{1}{B} \frac{\partial A}{\partial \alpha_{2}}+C_{1} \frac{P_{11}}{B^{2}} \frac{\partial B}{\partial \alpha_{2}} \frac{\partial A}{\partial \alpha_{2}}-C_{1} \frac{P_{11}}{B} \frac{\partial^{2} A}{\partial \alpha_{2}^{2}}+ \\
& +C_{1} \frac{P_{11}}{A} \frac{\partial^{2} B}{\partial \alpha_{1}^{2}}-C_{1} \frac{P_{11}}{A^{2}} \frac{\partial A}{\partial \alpha_{1}} \frac{\partial B}{\partial \alpha_{1}}+C_{1} \frac{P_{11}}{A} \frac{\partial B}{\partial \alpha_{1}}+C_{1} \frac{\partial P_{11}}{\partial \alpha_{1}} \frac{1}{A} \frac{\partial B}{\partial \alpha_{1}}-C_{1} \frac{\partial P_{11}}{\partial \alpha_{1}} \frac{B}{A^{2}} \frac{\partial A}{\partial \alpha_{1}}+ \\
& -C_{1} \frac{P_{11}}{A^{2}} \frac{\partial^{2} A}{\partial \alpha_{1}^{2}} B+C_{1} P_{11} \frac{B}{A^{2}} \frac{\partial^{2} A}{\partial \alpha_{1}^{2}}-N_{22} \frac{A B}{R_{2}}-C_{1} \frac{\partial P_{22}}{\partial \alpha_{1}} \frac{1}{A} \frac{\partial B}{\partial \alpha_{1}}+C_{1} \frac{P_{22}}{A^{2}} \frac{\partial A}{\partial \alpha_{1}} \frac{\partial B}{\partial \alpha_{1}}+ \\
& -C_{1} \frac{P_{22}}{A} \frac{\partial^{2} B}{\partial \alpha_{1}^{2}}+C_{1} \frac{P_{22}}{B} \frac{\partial^{2} A}{\partial \alpha_{2}^{2}}-C_{1} \frac{P_{22}}{B^{2}} \frac{\partial B}{\partial \alpha_{2}} \frac{\partial A}{\partial \alpha_{2}}+C_{1} \frac{P_{22}}{B} \frac{\partial A}{\partial \alpha_{2}}+C_{1} \frac{\partial P_{22}}{\partial \alpha_{2}} \frac{1}{B} \frac{\partial A}{\partial \alpha_{2}}+ \\
& -C_{1} \frac{\partial P_{22}}{\partial \alpha_{2}} \frac{A}{B^{2}} \frac{\partial B}{\partial \alpha_{2}}+C_{1} \frac{A}{B} \frac{\partial^{2} P_{22}}{\partial \alpha_{2}^{2}}-C_{1} \frac{P_{22}}{B^{2}} \frac{\partial^{2} B}{\partial \alpha_{2}^{2}} A+C_{1} P_{22} \frac{A}{B^{2}} \frac{\partial^{2} B}{\partial \alpha_{2}^{2}}+2 C_{1} \frac{\partial^{2} P_{12}}{\partial \alpha_{1} \partial \alpha_{2}}+ \\
& -2 C_{1} \frac{\partial P_{21}}{\partial \alpha_{2}} \frac{1}{B} \frac{\partial B}{\partial \alpha_{1}}+2 C_{1} \frac{P_{21}}{B^{2}} \frac{\partial B}{\partial \alpha_{2}} \frac{\partial B}{\partial \alpha_{1}}-2 C_{1} \frac{P_{21}}{B} \frac{\partial^{2} B}{\partial \alpha_{1} \partial \alpha_{2}}-2 C_{1} \frac{\partial P_{12}}{\partial \alpha_{1}} \frac{1}{A} \frac{\partial A}{\partial \alpha_{2}}+ \\
& +2 C_{1} \frac{P_{12}}{A^{2}} \frac{\partial A}{\partial \alpha_{1}} \frac{\partial A}{\partial \alpha_{2}}-2 C_{1} \frac{P_{12}}{A} \frac{\partial^{2} A}{\partial \alpha_{2} \partial \alpha_{1}}+\frac{\partial\left(Q_{13} B\right)}{\partial \alpha_{1}}-C_{2} \frac{\partial\left(R_{13} B\right)}{\partial \alpha_{1}}+\frac{\partial\left(Q_{23} A\right)}{\partial \alpha_{2}}+ \\
& -C_{2} \frac{\partial\left(R_{23} A\right)}{\partial \alpha_{2}}+q_{3} A B=0 \\
& \delta \varphi_{1}: \frac{\partial\left(M_{11} B\right)}{\partial \alpha_{1}}-C_{1} \frac{\partial\left(P_{11} B\right)}{\partial \alpha_{1}}-M_{22} \frac{\partial B}{\partial \alpha_{1}}+\frac{\partial\left(M_{21} A\right)}{\partial \alpha_{2}}+M_{12} \frac{\partial A}{\partial \alpha_{2}}+C_{1} P_{22} \frac{\partial B}{\partial \alpha_{1}}+ \\
& -C_{1} \frac{\partial\left(P_{21} A\right)}{\partial \alpha_{2}}-C_{1} P_{12} \frac{\partial A}{\partial \alpha_{2}}-Q_{13} A B+C_{2} R_{13} A B=0
\end{aligned}
$$




$$
\begin{aligned}
& \delta \varphi_{2}: \frac{\partial\left(M_{22} A\right)}{\partial \alpha_{2}}-C_{1} \frac{\partial\left(P_{22} A\right)}{\partial \alpha_{2}}-M_{11} \frac{\partial A}{\partial \alpha_{2}}+\frac{\partial\left(M_{12} B\right)}{\partial \alpha_{1}}+M_{21} \frac{\partial B}{\partial \alpha_{1}}+C_{1} P_{11} \frac{\partial A}{\partial \alpha_{2}}+ \\
& -C_{1} \frac{\partial\left(P_{12} B\right)}{\partial \alpha_{1}}-C_{1} P_{21} \frac{\partial B}{\partial \alpha_{1}}-Q_{23} A B+C_{2} R_{23} A B=0
\end{aligned}
$$

where $C_{1}=4 / 3 h^{2}$ and $C_{2}=4 / h^{2}$.

\section{Application of the higher-order theory to axisymmetric circular cylindrical shell of revolution}

Now let us cast the system of equations generated into a form appropriate for circular cylindrical shells of revolution.

The fundamental form of the reference surface is given by Soedel [13] as:

$$
(d s)^{2}=(d x)^{2}+a^{2}(d \theta)^{2}
$$

with its Lame parameters upon comparison with the expression in Eq.(2.1) obtained as

$$
\begin{aligned}
& A=1, \quad B=a=\text { const. }, \quad \text { with } \alpha_{1}=x, \quad \alpha_{2}=\theta, \\
& R_{1}=R_{x}=\infty, \quad R_{2}=R_{\theta}=a=\text { const } ., \quad 1 / R_{x}=0 .
\end{aligned}
$$

Note: the subscripts $1,2,3$ are replaced by $x, \theta$ and $z$, respectively.

\subsection{Governing differential equations of static equilibrium for circular cylindrical shell}

The substitution of Eq.(3.2) into the equations of equilibrium, Eqs (2.49)-(2.53), results in the governing partial differential equations of static equilibrium for circular cylindrical shells of revolution.

$$
\begin{aligned}
& \frac{\partial N_{x x}}{\partial x}+\frac{1}{a} \frac{\partial N_{\theta x}}{\partial \theta}+q_{x}=0, \\
& \frac{\partial N_{x \theta}}{\partial x}+\frac{1}{a} \frac{\partial N_{\theta \theta}}{\partial \theta}+q_{\theta}=0, \\
& C_{1} \frac{\partial^{2} P_{x x}}{\partial x^{2}}-\frac{N_{\theta \theta}}{a}+C_{1} \frac{\partial^{2} P_{\theta \theta}}{\partial \theta^{2}} \frac{1}{a^{2}}+2 C_{1} \frac{\partial^{2} P_{x \theta}}{\partial x \partial \theta} \frac{1}{a}+ \\
& +\frac{\partial Q_{x z}}{\partial x}-C_{2} \frac{\partial R_{x z}}{\partial x}+\frac{1}{a} \frac{\partial Q_{\theta z}}{\partial \theta}-C_{2} \frac{\partial R_{\theta z}}{\partial \theta} \frac{1}{a}+q_{z}=0, \\
& \frac{\partial M_{x x}}{\partial x}-C_{1} \frac{\partial P_{x x}}{\partial x}+\frac{1}{a} \frac{\partial M_{\theta x}}{\partial \theta}-C_{1} \frac{\partial P_{\theta x}}{\partial \theta} \frac{1}{a}-Q_{x z}+C_{2} R_{x z}=0, \\
& \frac{\partial M_{x \theta}}{\partial x}-C_{1} \frac{\partial P_{\theta \theta}}{\partial \theta} \frac{1}{a}+\frac{1}{a} \frac{\partial M_{\theta \theta}}{\partial \theta}-C_{1} \frac{\partial P_{x \theta}}{\partial x}-Q_{\theta z}+C_{2} R_{\theta z}=0 .
\end{aligned}
$$




\subsection{Strain components}

The strain components for an axisymmetric circular cylindrical shell of revolution are obtained by the substitution of Eq.(3.2) into the strain components, Eq.(2.13)

$$
\begin{aligned}
& \varepsilon_{x x}^{(0)}=\frac{\partial u}{\partial x}, \quad \kappa_{x x}^{(0)}=\frac{\partial \varphi_{x}}{\partial x}, \quad \kappa_{x x}^{(2)}=-C_{1}\left(\frac{\partial \varphi_{x}}{\partial x}+\frac{\partial^{2} w}{\partial x^{2}}\right), \quad \varepsilon_{\theta \theta}^{(0)}=\frac{1}{a}\left(\frac{\partial v}{\partial \theta}+w\right) \\
& \kappa_{\theta \theta}^{(0)}=\frac{1}{a} \frac{\partial \varphi_{\theta}}{\partial \theta}, \quad \kappa_{\theta \theta}^{(2)}=-C_{1}\left(\frac{1}{a} \frac{\partial \varphi_{\theta}}{\partial \theta}+\frac{1}{a^{2}} \frac{\partial^{2} w}{\partial \theta^{2}}\right), \quad \varepsilon_{x \theta}^{(0)}=\frac{1}{a} \frac{\partial u}{\partial \theta}+\frac{\partial v}{\partial x} \\
& \kappa_{x \theta}^{(0)}=\frac{1}{a} \frac{\partial \varphi_{x}}{\partial \theta}+\frac{\partial \varphi_{\theta}}{\partial x}, \quad \kappa_{x \theta}^{(2)}=-C_{1}\left(\frac{1}{a} \frac{\partial \varphi_{x}}{\partial \theta}+\frac{\partial \varphi_{\theta}}{\partial x}+\frac{2}{a} \frac{\partial^{2} w}{\partial x \partial \theta}\right) \\
& \varepsilon_{x z}^{(0)}=\varphi_{x}+\frac{\partial w}{\partial x}, \quad \kappa_{x z}^{(l)}=-C_{2}\left(\varphi_{x}+\frac{\partial w}{\partial x}\right), \\
& \varepsilon_{\theta z}^{(0)}=\varphi_{\theta}+\frac{1}{a} \frac{\partial w}{\partial \theta}, \quad \kappa_{\theta z}^{(l)}=-C_{2}\left(\varphi_{\theta}+\frac{1}{a} \frac{\partial w}{\partial \theta}\right) .
\end{aligned}
$$

\subsection{Elastic laws}

The stress resultant - displacement relations (elastic laws) for the circular cylindrical shell are obtained by the substitution of the strain components Eq.(3.8) into Eqs (2.21)-(2.33).

$$
\begin{aligned}
& N_{x x}=K\left[\frac{\partial u}{\partial x}+\frac{\mu}{a}\left(\frac{\partial v}{\partial \theta}+w\right)\right], \\
& N_{\theta \theta}=K\left[\mu \frac{\partial u}{\partial x}+\frac{1}{a}\left(\frac{\partial v}{\partial \theta}+w\right)\right], \\
& N_{x \theta}=\frac{K(1-\mu)}{2}\left[\frac{1}{a} \frac{\partial u}{\partial \theta}+\frac{\partial v}{\partial x}\right], \\
& M_{x x}=D\left[\frac{\partial \varphi_{x}}{\partial x}+\frac{\mu}{a} \frac{\partial \varphi_{\theta}}{\partial \theta}\right]-C_{1} F\left[\frac{\partial \varphi_{x}}{\partial x}+\frac{\partial^{2} w}{\partial x^{2}}+\frac{\mu}{a}\left(\frac{\partial \varphi_{\theta}}{\partial \theta}+\frac{1}{a} \frac{\partial^{2} w}{\partial \theta^{2}}\right)\right], \\
& M_{\theta \theta}=D\left[\mu \frac{\partial \varphi_{x}}{\partial x}+\frac{1}{a} \frac{\partial \varphi_{\theta}}{\partial \theta}\right]-C_{1} F\left[\mu\left(\frac{\partial \varphi_{x}}{\partial x}+\frac{\partial^{2} w}{\partial x^{2}}\right)+\frac{1}{a}\left(\frac{\partial \varphi_{\theta}}{\partial \theta}+\frac{1}{a} \frac{\partial^{2} w}{\partial \theta^{2}}\right)\right], \\
& M_{x \theta}=\frac{D(1-\mu)}{2}\left[\frac{\partial \varphi_{\theta}}{\partial x}+\frac{1}{a} \frac{\partial \varphi_{x}}{\partial \theta}\right]-C_{1} \frac{F(1-\mu)}{2}\left[\frac{1}{a} \frac{\partial \varphi_{x}}{\partial \theta}+\frac{\partial \varphi_{\theta}}{\partial x}+\frac{2}{a} \frac{\partial^{2} w}{\partial x \partial \theta}\right],
\end{aligned}
$$




$$
\begin{aligned}
& P_{x x}=F\left[\frac{\partial \varphi_{x}}{\partial x}+\frac{\mu}{a} \frac{\partial \varphi_{\theta}}{\partial \theta}\right]-C_{1} H\left[\frac{\partial \varphi_{x}}{\partial x}+\frac{\partial^{2} w}{\partial x^{2}}+\frac{\mu}{a}\left(\frac{\partial \varphi_{\theta}}{\partial \theta}+\frac{1}{a} \frac{\partial^{2} w}{\partial \theta^{2}}\right)\right], \\
& P_{x \theta}=\frac{F(1-\mu)}{2}\left[\frac{\partial \varphi_{\theta}}{\partial x}+\frac{1}{a} \frac{\partial \varphi_{x}}{\partial \theta}\right]-C_{1} \frac{H(1-\mu)}{2}\left[\frac{1}{a} \frac{\partial \varphi_{x}}{\partial \theta}+\frac{\partial \varphi_{\theta}}{\partial x}+\frac{2}{a} \frac{\partial^{2} w}{\partial x \partial \theta}\right] \\
& P_{\theta \theta}=F\left[\mu \frac{\partial \varphi_{x}}{\partial x}+\frac{1}{a} \frac{\partial \varphi_{\theta}}{\partial \theta}\right]-C_{1} H\left[\mu\left(\frac{\partial \varphi_{x}}{\partial x}+\frac{\partial^{2} w}{\partial x^{2}}\right)+\frac{1}{a}\left(\frac{\partial \varphi_{\theta}}{\partial \theta}+\frac{1}{a} \frac{\partial^{2} w}{\partial \theta^{2}}\right)\right], \\
& Q_{x z}=\frac{K(1-\mu)}{2}\left[\varphi_{x}+\frac{\partial w}{\partial x}\right]-C_{2} \frac{D(1-\mu)}{2}\left[\varphi_{x}+\frac{\partial w}{\partial x}\right] \\
& Q_{\theta z}=\frac{K(1-\mu)}{2}\left[\varphi_{\theta}+\frac{1}{a} \frac{\partial w}{\partial \theta}\right]-C_{2} \frac{D(1-\mu)}{2}\left[\varphi_{\theta}+\frac{1}{a} \frac{\partial w}{\partial \theta}\right] \\
& R_{x z}=\frac{D(1-\mu)}{2}\left[\varphi_{x}+\frac{\partial w}{\partial x}\right]-C_{2} \frac{F(1-\mu)}{2}\left[\varphi_{x}+\frac{\partial w}{\partial x}\right] \\
& R_{\theta z}=\frac{D(1-\mu)}{2}\left[\varphi_{\theta}+\frac{1}{a} \frac{\partial w}{\partial \theta}\right]-C_{2} \frac{F(1-\mu)}{2}\left[\varphi_{\theta}+\frac{1}{a} \frac{\partial w}{\partial \theta}\right] .
\end{aligned}
$$

\subsection{Equations of static equilibrium expressed in terms of displacements}

By using the stress resultant - displacement relations Eqs (3.9)-(3.21), the static equations of equilibrium Eqs (3.3)-(3.7) are expressed in terms of the displacements $u, v, w, \varphi_{x}$ and $\varphi_{\theta}$ as follows:

$$
\begin{aligned}
& \frac{\partial^{2} u}{\partial x^{2}}+\left(\frac{1-\mu}{2 a^{2}}\right) \frac{\partial^{2} u}{\partial \theta^{2}}+\left(\frac{1+\mu}{2 a^{2}}\right) \frac{\partial^{2} v}{\partial \theta \partial x}+\frac{\mu}{a} \frac{\partial w}{\partial x}=-\frac{q_{x}\left(1-\mu^{2}\right)}{E h} \\
& \frac{\partial^{2} u}{\partial x \partial \theta}\left(\frac{1+\mu}{2 a^{2}}\right)+\left(\frac{1-\mu}{2}\right) \frac{\partial^{2} v}{\partial x^{2}}+\frac{1}{a^{2}} \frac{\partial^{2} v}{\partial \theta^{2}}+\frac{1}{a^{2}} \frac{\partial w}{\partial \theta}=-\frac{q_{\theta}\left(1-\mu^{2}\right)}{E h} \\
& \frac{h^{2}}{60 a}\left(\frac{\mu}{a} \frac{\partial^{3} \varphi_{x}}{\partial x \partial \theta^{2}}+\frac{1}{a^{2}} \frac{\partial^{3} \varphi_{\theta}}{\partial \theta^{3}}\right)-\frac{h^{2}}{252 a}\left(\frac{\mu}{a} \frac{\partial^{3} \varphi_{x}}{\partial x \partial \theta^{2}}+\frac{\mu}{a} \frac{\partial^{4} w}{\partial \theta^{2} \partial x^{2}}+\frac{1}{a^{2}} \frac{\partial^{3} \varphi_{\theta}}{\partial \theta^{3}}+\frac{1}{a^{3}} \frac{\partial^{4} w}{\partial \theta^{4}}\right)+ \\
& +\frac{h^{2}(1-\mu)}{60}\left(\frac{\partial^{3} \varphi_{x}}{\partial x \partial \theta^{2}} \frac{1}{a^{2}}+\frac{\partial^{3} \varphi_{\theta}}{\partial \theta \partial x^{2}} \frac{1}{a}\right)-\frac{h^{2}(1-\mu)}{252}\left(\frac{\partial^{3} \varphi_{x}}{\partial x \partial \theta^{2}} \frac{1}{a^{2}}+\frac{\partial^{3} \varphi_{\theta}}{\partial \theta \partial x^{2}} \frac{1}{a}+\frac{2}{a^{2}} \frac{\partial^{4} w}{\partial x^{2} \partial \theta^{2}}\right)+ \\
& +\frac{4(1-\mu)}{15}\left(\frac{\partial \varphi_{x}}{\partial x}+\frac{\partial^{2} w}{\partial x^{2}}+\frac{1}{a} \frac{\partial \varphi_{\theta}}{\partial \theta}+\frac{1}{a^{2}} \frac{\partial^{2} w}{\partial \theta^{2}}\right)-\left(\frac{1}{a^{2}} \frac{\partial v}{\partial \theta}+\frac{w}{a^{2}}+\frac{\mu}{a} \frac{\partial u}{\partial x}\right)+ \\
& +\frac{h^{2}}{60}\left(\frac{\partial^{3} \varphi_{x}}{\partial x^{3}}+\frac{\mu}{a} \frac{\partial^{3} \varphi_{\theta}}{\partial \theta \partial x^{2}}\right)-\frac{h^{2}}{252}\left(\frac{\partial^{3} \varphi_{x}}{\partial x^{3}}+\frac{\partial^{4} w}{\partial x^{4}}+\frac{\mu}{a} \frac{\partial^{3} \varphi_{\theta}}{\partial \theta \partial x^{2}}+\frac{\mu}{a^{2}} \frac{\partial^{4} w}{\partial \theta^{2} \partial x^{2}}\right)=-\frac{q_{z}\left(1-\mu^{2}\right)}{E h}
\end{aligned}
$$




$$
\begin{aligned}
& \frac{h^{2}}{12}\left(\frac{\partial^{2} \varphi_{x}}{\partial x^{2}}+\frac{\mu}{a} \frac{\partial^{2} \varphi_{\theta}}{\partial \theta \partial x}\right)-\frac{h^{2}}{60}\left(2 \frac{\partial^{2} \varphi_{x}}{\partial x^{2}}+\frac{\partial^{3} w}{\partial x^{3}}+\frac{2 \mu}{a} \frac{\partial^{2} \varphi_{\theta}}{\partial \theta \partial x}+\frac{\mu}{a^{2}} \frac{\partial^{3} w}{\partial \theta^{2} \partial x}\right)+ \\
& +\frac{h^{2}}{252}\left(\frac{\partial^{2} \varphi_{x}}{\partial x^{2}} \frac{\partial^{3} w}{\partial x^{3}}+\frac{\mu}{a} \frac{\partial^{2} \varphi_{\theta}}{\partial \theta \partial x}+\frac{\mu}{a^{2}} \frac{\partial^{3} w}{\partial \theta^{2} \partial x}\right)+\frac{h^{2}(1-\mu)}{24 a}\left(\frac{1}{a} \frac{\partial^{2} \varphi_{x}}{\partial \theta^{2}}+\frac{\partial^{2} \varphi_{\theta}}{\partial \theta \partial x}\right)+ \\
& -\frac{h^{2}(1-\mu)}{120 a}\left(\frac{2}{a} \frac{\partial^{2} \varphi_{x}}{\partial \theta^{2}}+2 \frac{\partial^{2} \varphi_{\theta}}{\partial \theta \partial x}+\frac{2}{a} \frac{\partial^{3} w}{\partial \theta^{2} \partial x}\right)+\frac{h^{2}(1-\mu)}{504 a}\left(\frac{1}{a} \frac{\partial^{2} \varphi_{x}}{\partial \theta^{2}}+\frac{\partial^{2} \varphi_{\theta}}{\partial \theta \partial x}+\right. \\
& \left.+\frac{2}{a} \frac{\partial^{3} w}{\partial \theta^{2} \partial x}\right)-\frac{4(1-\mu)}{15}\left(\varphi_{x}+\frac{\partial w}{\partial x}\right)=0, \\
& \frac{h^{2}}{12 a}\left(\mu \frac{\partial^{2} \varphi_{x}}{\partial x \partial \theta}+\frac{1}{a} \frac{\partial^{2} \varphi_{\theta}}{\partial \theta^{2}}\right)-\frac{h^{2}}{60 a}\left(2 \mu \frac{\partial^{2} \varphi_{x}}{\partial x \partial \theta}+\mu \frac{\partial^{3} w}{\partial x^{2} \partial \theta}+\frac{2}{a} \frac{\partial^{2} \varphi_{\theta}}{\partial \theta^{2}}+\frac{1}{a^{2}} \frac{\partial^{3} w}{\partial \theta^{3}}\right)+ \\
& \frac{h^{2}}{252 a}\left(\mu \frac{\partial^{2} \varphi_{x}}{\partial x \partial \theta}+\mu \frac{\partial^{3} w}{\partial x^{2} \partial \theta}+\frac{1}{a} \frac{\partial^{2} \varphi_{\theta}}{\partial \theta^{2}}+\frac{1}{a^{2}} \frac{\partial^{3} w}{\partial \theta^{3}}\right)+\frac{h^{2}(1-\mu)}{24}\left(\frac{1}{a} \frac{\partial^{2} \varphi_{x}}{\partial x \partial \theta}+\frac{\partial^{2} \varphi_{\theta}}{\partial x^{2}}\right)+ \\
& -\frac{h^{2}(1-\mu)}{120}\left(\frac{2}{a} \frac{\partial^{2} \varphi_{x}}{\partial \theta \partial x}+2 \frac{\partial^{2} \varphi_{\theta}}{\partial x^{2}}+\frac{2}{a} \frac{\partial^{3} w}{\partial \theta \partial x^{2}}\right)+\frac{h^{2}(1-\mu)}{504}\left(\frac{1}{a} \frac{\partial^{2} \varphi_{x}}{\partial \theta \partial x}+\frac{\partial^{2} \varphi_{\theta}}{\partial x^{2}}+\right. \\
& \left.+\frac{2}{a} \frac{\partial^{3} w}{\partial \theta \partial x^{2}}\right)-\frac{4(1-\mu)}{15}\left(\varphi_{\theta}+\frac{\partial w}{\partial \theta} \frac{1}{a}\right)=0 .
\end{aligned}
$$

\subsection{Solution for a circular cylindrical shell of revolution with simply supported ends}

Consider a circular cylindrical shell under the influence of external loading with the ends simply supported at its edges. The imposed boundary conditions at ends $x=0, x=l$ are:

$$
N_{x x}=M_{x x}=P_{x x}=v=w=0 .
$$

If the applied load is expanded in a Fourier series given as:

$$
q_{z}=\sum_{m}^{\infty} \sum_{n}^{\infty} q_{m n} \cos n \theta \sin \frac{m \pi x}{l}, \quad q_{x}=q_{\theta}=0 .
$$

Uniform and sinusoidal loads are to be considered:

- with the cylinder filled to capacity (Fig. 2), the coefficients are given as [16]:

$$
q_{m n}=0, \quad q_{m o}=\frac{4 q d}{m \pi}, \quad q_{m l}=\frac{4 q a}{m \pi} \quad(m=1,3, \ldots . .),
$$

- sinusoidal loading

$$
q_{z}=q \cos n \theta \sin \frac{m \pi x}{l}, \quad \text { and } \quad m=1, \quad n=4 \quad \text { and } \quad q_{14}=q
$$




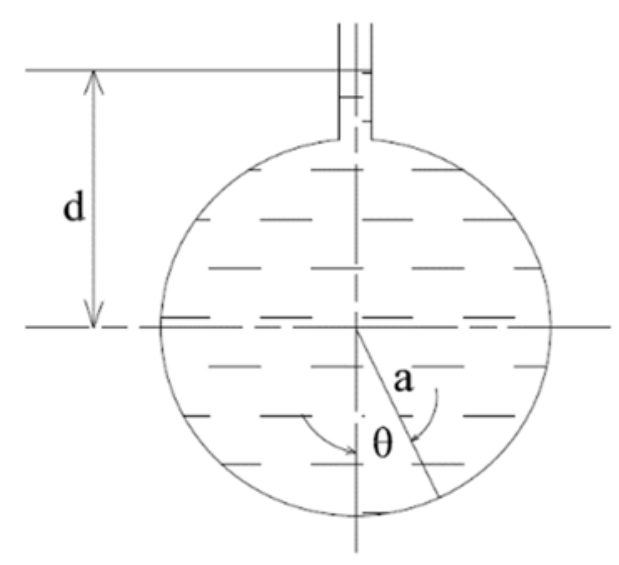

Fig.2. A circular cylinder filled to capacity [16].

The Navier type solutions are introduced as

$$
\begin{aligned}
& u=\sum_{m=1}^{\infty} \sum_{n=0}^{\infty} A_{m n} \cos \frac{m \pi x}{l} \cos n \theta, \\
& v=\sum_{m=1}^{\infty} \sum_{n=0}^{\infty} B_{m n} \sin \frac{m \pi x}{l} \sin n \theta, \\
& w=\sum_{m=1}^{\infty} \sum_{n=0}^{\infty} C_{m n} \sin \frac{m \pi x}{l} \cos n \theta, \\
& \varphi_{x}=\sum_{m=1}^{\infty} \sum_{n=0}^{\infty} D_{m n} \cos \frac{m \pi x}{l} \cos n \theta, \\
& \varphi_{\theta}=\sum_{m=1}^{\infty} \sum_{n=0}^{\infty} E_{m n} \sin \frac{m \pi x}{l} \sin n \theta .
\end{aligned}
$$

Substitution of Eqs (4.3)-(4.7) into the boundary conditions Eq.(4.1), results in the boundary conditions being satisfied by the adopted displacement functions at ends $x=0, x=l$.

Substituting Eqs (4.3)-(4.7) into the equation of equilibrium Eqs (3.22)-(3.26) results in the following form:

$$
\left[\begin{array}{ccccc}
Y_{11} & \mathrm{Y}_{12} & \mathrm{Y}_{13} & 0 & 0 \\
Y_{21} & \mathrm{Y}_{22} & \mathrm{Y}_{23} & 0 & 0 \\
Y_{31} & \mathrm{Y}_{32} & \mathrm{Y}_{33} & \mathrm{Y}_{34} & \mathrm{Y}_{35} \\
0 & 0 & \mathrm{Y}_{43} & \mathrm{Y}_{44} & \mathrm{Y}_{45} \\
0 & 0 & \mathrm{Y}_{53} & \mathrm{Y}_{54} & \mathrm{Y}_{55}
\end{array}\right]\left\{\begin{array}{l}
A_{m n} \\
B_{m n} \\
C_{m n} \\
D_{m n} \\
E_{m n}
\end{array}\right\}=\left\{\begin{array}{l}
0 \\
0 \\
-\frac{q_{m n}\left(1-\mu^{2}\right)}{E h} \\
0 \\
0
\end{array}\right\} .
$$


The coefficients of the matrix $Y_{i j}(i, j=1,2, \ldots, 5)$ are given below:

$$
\begin{aligned}
& Y_{11}=-\left[\left(\frac{m \pi}{l}\right)^{2}+n^{2} \frac{1-\mu}{2 a^{2}}\right], \quad Y_{12}=Y_{21}=n \frac{m \pi}{l} \frac{1+\mu}{2 a}, \quad Y_{13}=Y_{31}=\frac{\mu}{a} \frac{m \pi}{l}, \\
& Y_{22}=-\left[\frac{1-\mu}{2}\left(\frac{m \pi}{l}\right)^{2}+\frac{n^{2}}{a^{2}}\right], \quad Y_{23}=Y_{32}=-\frac{n}{a^{2}}, \\
& Y_{33}=-\frac{4(1-\mu)}{15}\left[\left(\frac{m \pi}{l}\right)^{2}+\frac{n^{2}}{a^{2}}\right]-\frac{1}{a^{2}}-\frac{h^{2}}{252}\left(\frac{m \pi}{l}\right)^{4}-\frac{h^{2}}{252} \frac{n^{4}}{a^{4}}-\frac{h^{2}}{126}\left(\frac{m \pi}{l}\right)^{2} \frac{n^{2}}{a^{2}}, \\
& Y_{34}=Y_{43}=-\frac{4(1-\mu)}{15} \frac{m \pi}{l}+\frac{4 h^{2}}{315}\left[\left(\frac{m \pi}{l}\right)^{3}+\frac{n^{2}}{a^{2}} \frac{m \pi}{l}\right], \\
& Y_{35}=Y_{53}=\frac{4(1-\mu)}{15} \frac{n}{a}-\frac{4 h^{2}}{315}\left[\left(\frac{m \pi}{l}\right)^{2} \frac{n}{a}+\frac{n^{3}}{a^{3}}\right], \\
& Y_{44}=-\frac{4(1-\mu)}{15}-\frac{17 h^{2}}{315}\left(\frac{m \pi}{l}\right)^{2}-\frac{17 h^{2}(1-\mu)}{630} \frac{n^{2}}{a^{2}}, \quad Y_{45}=Y_{45}=\frac{17 h^{2}}{630} \frac{n}{a} \frac{m \pi}{l}(1+\mu), \\
& Y_{55}=-\frac{4(1-\mu)}{15}-\frac{17 h^{2}}{315} \frac{n^{2}}{a^{2}}-\frac{17 h^{2}(1-\mu)}{630}\left(\frac{m \pi}{l}\right)^{2} .
\end{aligned}
$$

From the above coefficients $Y_{i j}(i, j=1,2, \ldots, 5)$ one can easily see that the matrix, Eq.(4.8), is a symmetric one, which points to the displacement being symmetric upholding Maxwell-Betti's theorem of reciprocity.

\section{Results and discussion}

The results obtained for the displacements and stresses are presented for the present solution which accounts for the transverse shear deformation. Comparison is made to the results obtained from the Kirchhoff - Love first approximation shell theory mostly known as the classical shell theory (CST) which ignores the transverse shear effects, for selected values of the mean-radius of curvature to thickness ratios $S=a / h$ at varying shell length to mean-radius of curvature $l / a$ ratios. The CST numerical results were generated based on the first approximation shell equations presented in the work of Soedel [13].

Case 1: Consider a simply supported cylindrical tank of elastic modulus $E$ filled with liquid of specific weight $\gamma$ to capacity (uniformly distributed loading) using the first few terms $m=1,3,5$ and $n=0,1$ for selected values of the mean radius to thickness ratio $S=a / h$. The maximum transverse deflection, axial and tangential stresses are determined using the following parameters: $a=d=50 \mathrm{~cm}, l=25 \mathrm{~cm}, \mu=0.3$. The results are presented in terms of the following non - dimensional definitions as in Eq.(5.1) 


$$
\bar{w}=\left(\frac{10 E h^{3}}{\gamma a^{4}}\right) w, \quad \overline{\sigma_{x}}=\left(\frac{10 h^{2}}{\gamma a^{2}}\right) \sigma_{x}, \quad \overline{\sigma_{\theta}}=\left(\frac{10 h^{2}}{\gamma a^{2}}\right) \sigma_{\theta} .
$$

The maximum values of the transverse deflection and internal force resultants are obtained at $x=l / 2, \theta=0$.

Table 1 presents the maximum deflection for the shell subjected to uniformly distributed loading (UDL). It can be observed that both theories have identical results with very little percentage variations for shells classified as thin $S \geq 20$. The difference in variations, however, becomes comparatively more significant with the continuous decrease in values of $S$ (thicker shells). For cylindrical shells subjected to UDL within the range $20>S \geq 4$, the percentage variations in transverse deflection are within $6.5 \%$ and $58 \%$ for the moderately thick case $(S=10)$ and very thick case $(S=4)$, respectively, with the shell length to mean-radius of curvature ratio $l / a=0.5$.

Table 1. Non-dimensional results for the maximum deflection, axial and tangential stresses for a simply supported circular cylindrical tank filled to capacity (UDL) for $l / a=0.5$.

\begin{tabular}{|c|c|c|c|c|c|c|c|c|c|}
\hline & \multicolumn{4}{|c|}{$\bar{w}$} & \multicolumn{3}{c|}{$\overline{\sigma_{x}}\left(\alpha_{3}=h / 2\right)$} & \multicolumn{3}{c|}{$\overline{\sigma_{\theta}}\left(\alpha_{3}=h / 2\right)$} \\
\hline $\boldsymbol{S}$ & $\begin{array}{c}\text { Present } \\
\text { study }\end{array}$ & $\mathbf{C S T}$ & $\% \boldsymbol{\Delta}$ & $\begin{array}{c}\text { Present } \\
\text { study }\end{array}$ & $\mathbf{C S T}$ & $\% \Delta$ & $\begin{array}{c}\text { Present } \\
\text { study }\end{array}$ & $\mathbf{C S T}$ & $\% \Delta$ \\
\hline $\mathbf{1 0 0 . 0 0}$ & 0.1115 & 0.1115 & - & 0.5163 & 0.5070 & +1.836 & 10.9713 & 10.9720 & -0.006 \\
\hline $\mathbf{8 3 . 3 3}$ & 0.1637 & 0.1638 & -0.058 & 1.1668 & 1.1563 & +0.907 & 13.5997 & 13.6046 & -0.036 \\
\hline $\mathbf{7 1 . 4 3}$ & 0.2257 & 0.2259 & -0.081 & 2.1362 & 2.1277 & +0.400 & 16.3171 & 16.3277 & -0.065 \\
\hline $\mathbf{6 2 . 5 0}$ & 0.2969 & 0.2972 & -0.099 & 3.3923 & 3.3896 & +0.080 & 19.0826 & 19.1003 & -0.093 \\
\hline $\mathbf{5 5 . 5 6}$ & 0.3768 & 0.3773 & -0.110 & 4.9018 & 4.9090 & -0.147 & 21.8672 & 21.8930 & -0.118 \\
\hline $\mathbf{5 0 . 0 0}$ & 0.4648 & 0.4653 & -0.118 & 6.6342 & 6.6560 & -0.328 & 24.6496 & 24.6842 & -0.140 \\
\hline $\mathbf{2 5 . 0 0}$ & 1.6542 & 1.6523 & +0.115 & 31.4798 & 31.9541 & -1.480 & 50.0178 & 50.1221 & -0.208 \\
\hline $\mathbf{2 0 . 0 0}$ & 2.3519 & 2.3404 & +0.495 & 45.9798 & 46.8953 & -1.952 & 60.0660 & 60.1293 & -0.105 \\
\hline $\mathbf{1 6 . 6 7}$ & 3.0509 & 3.0177 & +1.100 & 60.1751 & 61.6255 & -2.354 & 68.1957 & 68.1138 & +0.121 \\
\hline $\mathbf{1 2 . 5 0}$ & 4.3568 & 4.2275 & +3.060 & 85.3792 & 87.9360 & -2.910 & 79.5654 & 78.7955 & +0.976 \\
\hline $\mathbf{1 0 . 0 0}$ & 5.4997 & 5.1860 & +6.050 & 105.3820 & 108.7640 & -3.110 & 86.3211 & 84.3300 & +2.360 \\
\hline $\mathbf{7 . 1 4}$ & 7.4263 & 6.4581 & +15.000 & 132.8155 & 136.3670 & -2.604 & 92.9706 & 87.3552 & +6.420 \\
\hline $\mathbf{5 . 0 0}$ & 10.0393 & 7.4243 & +35.221 & 156.9600 & 157.2800 & -0.198 & 97.7240 & 85.1080 & +14.830 \\
\hline $\mathbf{4 . 0 0}$ & 12.3634 & 7.8292 & +57.908 & 170.8750 & 166.0212 & +2.920 & 101.3390 & 82.1814 & +23.311 \\
\hline
\end{tabular}

Case 2: Consider a simply supported circular cylindrical shell with elastic modulus $E$ under the influence of external sinusoidal transverse surface load $q$ for the fundamental mode $m=1$ and $n=4$ as found in works of (Di and Rothert, [8]; Amabili and Reddy, [17]). The displacements and stresses are determined for the selected length to mean radius of curvature ratios $l / a$ using the following parameters: $a=d=50 \mathrm{~cm}$; $l=25 \mathrm{~cm}, 125 \mathrm{~cm}, 250 \mathrm{~cm}$ and $500 \mathrm{~cm} ; \mu=0.3$.

The results are presented in terms of non-dimensional definitions as given in Di and Rothert [8] as follows;

$$
\begin{array}{ll}
\bar{w}=\left(\frac{10 E h^{3}}{q a^{4}}\right) w /\left(\cos n \theta \sin \frac{m \pi x}{l}\right), & \bar{u}=\left(\frac{10 E h^{2}}{q a^{3}}\right) u /\left(\cos n \theta \cos \frac{m \pi x}{l}\right), \\
\bar{v}=\left(\frac{10 E h^{2}}{q a^{3}}\right) v /\left(\cos n \theta \sin \frac{m \pi x}{l}\right), & \overline{\sigma_{x}}=\left(\frac{10 h^{2}}{q a^{2}}\right) \sigma_{x} /\left(\cos n \theta \sin \frac{m \pi x}{l}\right),
\end{array}
$$




$$
\begin{array}{ll}
\overline{\sigma_{\theta}}=\left(\frac{10 h^{2}}{q a^{2}}\right) \sigma_{\theta} /\left(\cos n \theta \sin \frac{m \pi x}{l}\right), & \overline{\sigma_{x \theta}}=\left(\frac{10 h^{2}}{q a^{2}}\right) \sigma_{x \theta} /\left(\sin n \theta \cos \frac{m \pi x}{l}\right), \\
\overline{\sigma_{x z}}=\left(\frac{10 h}{q a}\right) \sigma_{x Z} /\left(\cos n \theta \cos \frac{m \pi x}{l}\right), & \overline{\sigma_{\theta z}}=\left(\frac{10 h}{q a}\right) \sigma_{\theta Z} /\left(\sin n \theta \sin \frac{m \pi x}{l}\right) .
\end{array}
$$

(cont.5.2)

Tables 2, 3, 4 and 5 show the non-dimensional displacements for the shell subjected to sinusoidal distributed loading (SDL) with varying length to mean radius of curvature ratios $l / a=0.5,2.5,5$ and 10 for selected values of $S$. The results obtained likewise point to significant variations in obtained results of the displacements for the moderately thick to very thick case $(S=10,4)$. However, it is observed that discrepancies in results become less significant with a continuous increase in the ratios $l / a$, with variations in displacements for the very thick case $S=4$ recorded within $93 \%, 32 \%, 30 \%$ and $29 \%$ for $l / a=0.5,2.5,5$ and 10 , respectively.

Table 2. Non-dimensional displacements $\bar{w}, \bar{u}$ and $\bar{v}$ for a simply supported circular cylindrical shell under sinusoidal loading (SDL) for $l / a=0.5$.

\begin{tabular}{|l|c|c|c|c|c|c|c|c|c|}
\hline \multicolumn{3}{|c|}{$\bar{w}$} & \multicolumn{3}{c|}{$\bar{u}$} & \multicolumn{3}{|c|}{$\bar{v}$} \\
\hline $\boldsymbol{S}$ & $\begin{array}{c}\text { Present } \\
\text { Study }\end{array}$ & $\mathbf{C S T}$ & $\% \boldsymbol{\Delta}$ & $\begin{array}{c}\text { Present } \\
\text { study }\end{array}$ & $\mathbf{C S T}$ & $\% \boldsymbol{\Delta}$ & $\begin{array}{c}\text { Present } \\
\text { study }\end{array}$ & $\mathbf{C S T}$ & $\% \Delta$ \\
\hline $\mathbf{1 0 0}$ & 0.00187 & 0.00187 & & -0.00159 & -0.00159 & & -0.02597 & -0.02597 & \\
\hline $\mathbf{2 0}$ & 0.02111 & 0.02064 & +2.277 & -0.00358 & -0.00351 & +1.994 & -0.05861 & -0.05731 & +2.268 \\
\hline $\mathbf{1 0}$ & 0.03401 & 0.03008 & +13.065 & -0.00289 & -0.00255 & +13.330 & -0.04721 & -0.04175 & +13.077 \\
\hline $\mathbf{4}$ & 0.06644 & 0.03449 & +92.636 & -0.00225 & -0.00117 & +92.308 & -0.03688 & -0.01915 & +92.584 \\
\hline
\end{tabular}

Table 3. Non-dimensional displacements $\bar{w}, \bar{u}$ and $\bar{v}$ for a simply supported circular cylindrical shell under sinusoidal loading (SDL) for $l / a=2.5$.

\begin{tabular}{|l|c|c|c|c|c|c|c|c|c|}
\hline & \multicolumn{3}{|c|}{$\bar{w}$} & \multicolumn{3}{|c|}{$\bar{u}$} & \multicolumn{3}{|c|}{$\bar{v}$} \\
\hline $\mathbf{S}$ & $\begin{array}{c}\text { Present } \\
\text { Study }\end{array}$ & $\mathbf{C S T}$ & $\% \Delta$ & $\begin{array}{c}\text { Present } \\
\text { study }\end{array}$ & $\mathbf{C S T}$ & $\% \Delta$ & $\begin{array}{c}\text { Present } \\
\text { study }\end{array}$ & $\mathbf{C S T}$ & $\% \Delta$ \\
\hline $\mathbf{1 0 0}$ & 0.0903 & 0.09166 & -1.484 & -0.57012 & -0.57861 & -1.467 & -2.2946 & -2.32922 & -1.486 \\
\hline $\mathbf{2 0}$ & 0.32004 & 0.31715 & +0.911 & -0.40414 & -0.40040 & +0.934 & -1.62656 & -1.61184 & +0.913 \\
\hline $\mathbf{1 0}$ & 0.36010 & 0.34356 & +4.814 & -0.22736 & -0.21687 & +4.837 & -0.91506 & -0.87302 & +4.815 \\
\hline $\mathbf{4}$ & 0.46106 & 0.35177 & +31.069 & -0.11645 & -0.08882 & +31.108 & -0.46863 & -0.35755 & +31.067 \\
\hline
\end{tabular}

Table 4. Non-dimensional displacements $\bar{w}, \bar{u}$ and $\bar{v}$ for a simply supported circular cylindrical shell under

\begin{tabular}{|c|c|c|c|c|c|c|c|c|c|}
\hline & \multicolumn{3}{|c|}{$\bar{w}$} & \multicolumn{3}{|c|}{$\bar{u}$} & \multicolumn{3}{|c|}{$\bar{v}$} \\
\hline$S$ & $\begin{array}{l}\text { Present } \\
\text { Study }\end{array}$ & CST & $\% \Delta$ & $\begin{array}{c}\text { Present } \\
\text { study }\end{array}$ & CST & $\% \Delta$ & $\begin{array}{c}\text { Present } \\
\text { study }\end{array}$ & CST & $\% \Delta$ \\
\hline 100 & 0.32876 & 0.32912 & -0.109 & -1.22050 & -1.22230 & -0.147 & -8.27210 & -8.28119 & -0.110 \\
\hline 20 & 0.40712 & 0.40250 & +1.148 & -0.3023 & -0.29900 & +1.104 & -2.04875 & -2.02553 & +1.146 \\
\hline 10 & 0.42422 & 0.40532 & +4.663 & -0.15750 & -0.15053 & +4.630 & -1.06742 & -1.01987 & +4.662 \\
\hline 4 & 0.52452 & 0.40612 & +29.154 & -0.0779 & -0.06033 & +29.123 & -0.52788 & -0.40875 & +29.145 \\
\hline
\end{tabular}
sinusoidal loading (SDL) for $l / a=5$. 
Further observation of these discrepancies through varying length to radius of curvature ratios, points to a lessening of its severity with increasing length to radius ratios from $l / a=0.5-10$. An examination of the non-dimensional out of plane displacements (deflection) through the varying $l / a$ ratios shows a continual increase in the transverse deflection as the shell length increases for same values of $S$. This behavior can be associated with that of an elastic beam in which deflection is proportional to its span with its distributed loading, inertia and other material properties remaining constant.

Table 5. Non-dimensional displacements $\bar{w}, \bar{u}$ and $\bar{v}$ for a simply supported circular cylindrical shell under sinusoidal loading (SDL) for $l / a=10$.

\begin{tabular}{|c|c|c|c|c|c|c|c|c|c|}
\hline & \multicolumn{3}{|c|}{$\bar{w}$} & \multicolumn{3}{c|}{$\bar{u}$} & \multicolumn{3}{c|}{$\bar{v}$} \\
\hline $\boldsymbol{S}$ & $\begin{array}{c}\text { Present } \\
\text { Study }\end{array}$ & $\mathbf{C S T}$ & $\% \boldsymbol{\Delta}$ & $\begin{array}{c}\text { Present } \\
\text { study }\end{array}$ & $\mathbf{C S T}$ & $\% \boldsymbol{\Delta}$ & $\begin{array}{c}\text { Present } \\
\text { study }\end{array}$ & $\mathbf{C S T}$ & $\% \Delta$ \\
\hline $\mathbf{1 0 0}$ & 0.41515 & 0.41463 & +0.113 & -0.80365 & -0.80276 & +0.111 & -10.39740 & -10.38559 & +0.114 \\
\hline $\mathbf{2 0}$ & 0.42591 & 0.42108 & +1.147 & -0.16490 & -0.16303 & +1.148 & -2.13336 & -2.10916 & +1.148 \\
\hline $\mathbf{1 0}$ & 0.44063 & 0.42128 & +4.593 & -0.08530 & -0.08155 & +4.593 & -1.10354 & -1.05509 & +4.592 \\
\hline $\mathbf{4}$ & 0.54202 & 0.42134 & +28.643 & -0.04197 & -0.03263 & +28.624 & -0.54300 & -0.42209 & +28.646 \\
\hline
\end{tabular}

Table 6. Non-dimensional in-plane normal stresses $\overline{\sigma_{x}}, \overline{\sigma_{\theta}}$ and in-plane shearing stress $\overline{\sigma_{x \theta}}$ for a simply supported circular cylindrical shell under sinusoidal loading (SDL) for $l / a=0.5$.

\begin{tabular}{|c|l|l|l|l|l|l|l|l|l|}
\hline & \multicolumn{3}{|c|}{$\overline{\sigma_{x}}\left(\alpha_{3}= \pm h / 2\right)$} & \multicolumn{3}{c|}{$\overline{\sigma_{\theta}}\left(\alpha_{3}= \pm h / 2\right)$} & \multicolumn{3}{c|}{$\overline{\sigma_{x \theta}}\left(\alpha_{3}= \pm h / 2\right)$} \\
\hline $\boldsymbol{S}$ & $\begin{array}{c}\text { Present } \\
\text { Study }\end{array}$ & CST & $\% \Delta$ & $\begin{array}{c}\text { Present } \\
\text { Study }\end{array}$ & CST & $\% \Delta$ & $\begin{array}{c}\text { Present } \\
\text { study }\end{array}$ & CST & $\% \Delta$ \\
\hline $\mathbf{1 0 0}$ & 0.08382 & 0.08393 & -0.131 & 0.12331 & 0.12336 & -0.041 & -0.04227 & -0.04222 & +0.118 \\
& -0.00702 & -0.00712 & -1.404 & 0.06618 & 0.06611 & +0.106 & -0.07836 & -0.07840 & -0.051 \\
\hline $\mathbf{2 0}$ & 0.58361 & 0.58718 & -0.608 & 0.52633 & 0.52504 & +0.246 & 0.06132 & 0.06650 & -7.789 \\
& -0.41029 & -0.41764 & -1.760 & -0.09867 & -0.10682 & -7.630 & -0.33358 & -0.33273 & +0.255 \\
\hline $\mathbf{1 0}$ & 0.80262 & 0.79372 & +1.121 & 0.63307 & 0.61261 & +3.340 & 0.18150 & 0.19386 & -6.376 \\
& -0.66300 & -0.67022 & -1.077 & -0.28856 & -0.30796 & -6.300 & -0.40083 & -0.38780 & +3.360 \\
\hline \multirow{4}{*}{} & 1.00381 & 0.86769 & +15.688 & 0.73181 & 0.59769 & +22.44 & 0.29163 & 0.2890 & +0.910 \\
& -0.89469 & -0.81100 & +10.319 & -0.46254 & -45794 & +1.004 & -0.46294 & -0.37800 & +22.471 \\
\hline
\end{tabular}

The results of the non-dimensional in-plane normal and shearing stresses for the case of a UDL and SDL are presented in Tabs 1, 6, 7, 8 and 9. From the results obtained, it is realized that the variations in stresses are more subtle than these shown by the displacements, with percentage variations obtained for the very thick case $(S=4)$ within $24 \%, 7 \%, 6 \%$ and $5 \%$ for $l / a=0.5,2.5,5$ and 10 , respectively. However, for the moderately thick case $S=10$, variations in stresses are obtained within $7 \%, 2 \%, 1 \%$ and $0.8 \%$ for $l / a=0.5$, 2.5, 5 and 10, respectively, highlighting the ability of CST to estimate the in-plane normal and shearing stresses in thin to moderately thick shells within the engineering admissible error of $5 \%$ for $l / a$ ratios within $2.5-10$.

Generally, one can see that a rise in the shell's bending deformation as a consequence of an increase in the shell's length to mean-radius of curvature ratios $l / a$, results in diminishing transverse shearing effects on the displacements and stresses for same values of mean-radius to thickness ratios $S$. However the classical (first approximation) shell theory ultimately fails to predict acceptable values of the displacements even at sufficiently high $l / a$ ratios in thick shells due to the neglect of the transverse shear effects. 
Table 7. Non-dimensional in-plane normal stresses $\overline{\sigma_{x}}, \overline{\sigma_{\theta}}$ and in-plane shearing stress $\overline{\sigma_{x \theta}}$ for a simply supported circular cylindrical shell under sinusoidal loading (SDL) for $l / a=2.5$.

\begin{tabular}{|c|l|l|l|l|l|l|l|l|l|}
\hline & \multicolumn{3}{|c|}{$\overline{\sigma_{x}}\left(\alpha_{3}= \pm h / 2\right)$} & \multicolumn{3}{c|}{$\overline{\sigma_{\theta}}\left(\alpha_{3}= \pm h / 2\right)$} & \multicolumn{3}{c|}{$\overline{\sigma_{x \theta}}\left(\alpha_{3}= \pm h / 2\right)$} \\
\hline $\boldsymbol{S}$ & $\begin{array}{c}\text { Present } \\
\text { Study }\end{array}$ & CST & $\% \Delta$ & $\begin{array}{c}\text { Present } \\
\text { study }\end{array}$ & CST & $\% \Delta$ & $\begin{array}{c}\text { Present } \\
\text { study }\end{array}$ & CST & $\% \Delta$ \\
\hline $\mathbf{1 0 0}$ & 1.05448 & 1.07076 & -1.520 & 0.88925 & 0.90396 & -1.627 & -0.05760 & -0.05836 & -1.302 \\
& 0.42225 & 0.42803 & -1.350 & -0.74352 & -0.75584 & -1.630 & -0.40631 & -0.41287 & -1.589 \\
\hline $\mathbf{2 0}$ & 1.63269 & 1.63051 & +0.134 & 2.91646 & 2.92273 & -0.215 & 0.44741 & 0.45028 & -0.637 \\
& -0.58590 & -0.59334 & -1.254 & -2.81313 & -2.82023 & -0.252 & -0.77625 & -0.77636 & -0.014 \\
\hline $\mathbf{1 0}$ & 1.50570 & 1.48543 & +1.365 & 3.15633 & 3.13842 & +0.571 & 0.57538 & 0.57608 & -0.122 \\
& -0.91646 & -0.92362 & -0.775 & -3.09819 & -3.08279 & +0.500 & -0.76040 & -0.75269 & +1.024 \\
\hline $\mathbf{4}$ & 1.43956 & 1.34831 & +6.768 & 3.33913 & 3.19625 & +4.470 & 0.66444 & 0.64406 & +3.164 \\
& -1.13788 & -1.11825 & +1.755 & -3.30906 & -3.17375 & +4.263 & -0.75919 & -0.71644 & +5.967 \\
\hline
\end{tabular}

Table 8. Non-dimensional in-plane normal stresses $\overline{\sigma_{x}}, \overline{\sigma_{\theta}}$ and in-plane shearing stress $\overline{\sigma_{x \theta}}$ for a simply supported circular cylindrical shell under sinusoidal loading (SDL) for $l / a=5$.

\begin{tabular}{|c|l|l|l|l|l|l|l|l|l|}
\hline & \multicolumn{3}{|c|}{$\overline{\sigma_{x}}\left(\alpha_{3}= \pm h / 2\right)$} & \multicolumn{3}{c|}{$\overline{\sigma_{\theta}}\left(\alpha_{3}= \pm h / 2\right)$} & \multicolumn{3}{c|}{$\overline{\sigma_{x \theta}}\left(\alpha_{3}= \pm h / 2\right)$} \\
\hline \multirow{2}{*}{$\boldsymbol{S}$} & $\begin{array}{l}\text { Present } \\
\text { Study }\end{array}$ & CST & $\% \Delta$ & $\begin{array}{c}\text { Present } \\
\text { study }\end{array}$ & CST & $\% \Delta$ & $\begin{array}{c}\text { Present } \\
\text { study }\end{array}$ & CST & $\% \Delta$ \\
\hline \multirow{2}{*}{100} & 1.70995 & 1.71340 & -0.201 & 2.92732 & 2.93453 & -0.246 & 0.19606 & 0.19741 & -0.684 \\
& -0.16465 & -0.16589 & -0.747 & -2.88919 & -2.89655 & -0.254 & -0.43880 & -0.43904 & -0.055 \\
\hline \multirow{2}{*}{20} & 1.34132 & 1.33841 & +0.217 & 3.57282 & 3.57025 & +0.072 & 0.35939 & 0.35963 & -0.067 \\
& -0.95859 & -0.95990 & -1.760 & -3.56336 & -3.56097 & +0.067 & -0.41951 & -0.41873 & +0.186 \\
\hline \multirow{2}{*}{10} & 1.26453 & 1.25251 & +0.960 & 3.61674 & 3.59297 & +0.662 & 0.37881 & 0.37703 & +0.472 \\
& -1.06515 & -1.06193 & +0.303 & -3.61189 & -3.58829 & +0.658 & -0.41014 & -0.40679 & +0.824 \\
\hline \multirow{2}{*}{4} & 1.26381 & 1.19769 & +5.521 & 3.76964 & 3.59863 & +4.752 & 0.40356 & 0.38675 & +4.346 \\
& -1.16513 & -1.21310 & +3.908 & -3.76688 & -3.59675 & +4.730 & -0.41906 & -0.39863 & +5.125 \\
\hline
\end{tabular}

Table 9. Non-dimensional in-plane normal stresses $\overline{\sigma_{x}}, \overline{\sigma_{\theta}}$ and in-plane shearing stress $\overline{\sigma_{x \theta}}$ for a simply supported circular cylindrical shell under sinusoidal loading (SDL) for $l / a=10$.

\begin{tabular}{|c|c|c|c|c|c|c|c|c|c|}
\hline & \multicolumn{3}{|c|}{$\overline{\sigma_{x}}\left(\alpha_{3}= \pm h / 2\right)$} & \multicolumn{3}{|c|}{$\overline{\sigma_{\theta}}\left(\alpha_{3}= \pm h / 2\right)$} & \multicolumn{3}{|c|}{$\overline{\sigma_{x \theta}}\left(\alpha_{3}= \pm h / 2\right)$} \\
\hline$S$ & $\begin{array}{c}\text { Present } \\
\text { Study }\end{array}$ & CST & $\% \Delta$ & $\begin{array}{c}\text { Present } \\
\text { study }\end{array}$ & CST & $\% \Delta$ & $\begin{array}{c}\text { Present } \\
\text { study }\end{array}$ & CST & $\% \Delta$ \\
\hline 100 & $\begin{array}{c}1.36913 \\
-0.86319\end{array}$ & $\begin{array}{l}1.36915 \\
-.86377\end{array}$ & $\begin{array}{l}-0.001 \\
-0.067\end{array}$ & $\begin{array}{r}3.65388 \\
-3.65075\end{array}$ & $\begin{array}{c}3.65487 \\
-3.65171\end{array}$ & $\begin{array}{l}-0.027 \\
-0.026\end{array}$ & $\begin{array}{r}0.18050 \\
-0.22037\end{array}$ & $\begin{array}{r}0.18059 \\
-0.22037\end{array}$ & $\begin{array}{l}-0.050 \\
+0.001\end{array}$ \\
\hline 20 & $\begin{array}{r}1.18657 \\
-1.08277\end{array}$ & $\begin{array}{l}1.18500 \\
-.08236\end{array}$ & $\begin{array}{l}+0.132 \\
+0.038\end{array}$ & $\begin{array}{c}3.71320 \\
-3.71256\end{array}$ & $\begin{array}{c}3.70997 \\
-3.70933\end{array}$ & $\begin{array}{l}+0.087 \\
+0.087\end{array}$ & $\begin{array}{c}0.19967 \\
-0.20785\end{array}$ & $\begin{array}{c}0.19953 \\
-0.20761\end{array}$ & $\begin{array}{l}+0.070 \\
+0.115\end{array}$ \\
\hline 10 & $\begin{array}{r}1.16856 \\
-1.11486\end{array}$ & $\begin{array}{l}1.15990 \\
-.10856\end{array}$ & $\begin{array}{l}+0.746 \\
+0.569\end{array}$ & $\begin{array}{r}3.73608 \\
-3.73574\end{array}$ & $\begin{array}{c}3.71161 \\
-3.71128\end{array}$ & $\begin{array}{l}+0.659 \\
+0.659\end{array}$ & $\begin{array}{c}0.20291 \\
-0.20714\end{array}$ & $\begin{array}{c}0.20165 \\
-0.20569\end{array}$ & $\begin{array}{l}+0.622 \\
+0.705\end{array}$ \\
\hline 4 & $\begin{array}{c}1.20051 \\
-1.17410\end{array}$ & $\begin{array}{l}1.14465 \\
-.12410\end{array}$ & $\begin{array}{l}+4.880 \\
+4.447\end{array}$ & $\begin{array}{c}3.88516 \\
-3.88506\end{array}$ & $\begin{array}{c}3.71201 \\
-3.71188\end{array}$ & $\begin{array}{l}+4.665 \\
+4.666\end{array}$ & $\begin{array}{c}0.21217 \\
-0.21426\end{array}$ & $\begin{array}{c}0.20289 \\
-0.20451\end{array}$ & $\begin{array}{l}+4.574 \\
+4.767\end{array}$ \\
\hline
\end{tabular}


The non-dimensional transverse shearing stresses distribution for the present study presented in Tab.10. below depicts a parabolic (quadratic) distribution of the transverse shear stresses together with the condition of stress free boundary surfaces satisfied. This is in agreement with the predictions of the theory of elasticity and ensures that the need for shear correction coefficients is thereby obviated. The symmetric distribution of the transverse shearing stresses is as a result of the assumption of symmetry in transverse shear deformation while developing the present solution. This is done by adopting an odd function (cubic) expansion of the in-plane displacements (i.e. Eqs (2.2) and (2.3)), where first derivatives are of the form of even functions, hence the transverse shear symmetry about the shell middle surface.

Table 10. Thickness distribution of the non-dimensional transverse shearing stresses $\overline{\sigma_{x z}}, \overline{\sigma_{\theta z}}$ of the present study for a simply supported circular cylindrical shell under sinusoidal loading for $S=10$.

\begin{tabular}{|c|c|c|c|c|c|c|c|c|c|c|c|c|}
\hline \multicolumn{13}{|c|}{ Thickness coordinate $\alpha_{3}=r h$} \\
\hline \multicolumn{2}{|l|}{$r$} & $-1 / 2$ & $-1 / 3$ & $-1 / 4$ & $-1 / 5$ & $-1 / 6$ & 0 & $1 / 6$ & $1 / 5$ & $1 / 4$ & $1 / 3$ & $1 / 2$ \\
\hline$\frac{l}{-}=0.5$ & $\sigma_{x z}$ & 0 & 0.7815 & 1.0526 & 1.1781 & 1.2463 & 1.4013 & 1.2463 & 1.1781 & 1.0526 & 0.7815 & 0 \\
\hline$a$ & $\overline{\sigma_{\theta z}}$ & 0 & -0.4971 & -0.6670 & -0.7494 & -0.7927 & -0.8913 & -0.7927 & -0.7494 & -0.6670 & -0.4971 & 0 \\
\hline$\frac{l}{l}=2.5$ & $\sigma_{x z}$ & 0 & 0.5828 & 0.7849 & 0.8785 & 0.9294 & 1.0449 & 0.9294 & 0.8785 & 0.7849 & 0.5828 & 0 \\
\hline $\bar{a}^{-2.0}$ & $\overline{\sigma_{\theta z}}$ & 0 & -1.8418 & -2.4806 & -2.7765 & -2.9372 & -3.3024 & -2.9372 & -2.7765 & -2.4806 & -1.8418 & 0 \\
\hline$\frac{l}{-}=5$ & $\overline{\sigma_{x z}}$ & 0 & 0.3206 & 0.4318 & 0.4833 & 0.5112 & 0.5748 & 0.5112 & 0.4833 & 0.4318 & 0.3206 & 0 \\
\hline & $\overline{\sigma_{\theta z}}$ & 0 & -2.0301 & -2.7342 & -3.0603 & -3.2374 & -3.6400 & -3.2374 & -3.0603 & -2.7342 & -2.0301 & 0 \\
\hline$\underline{l}=10$ & $\sigma_{x z}$ & 0 & 0.1628 & 0.2192 & 0.2454 & 0.2596 & 0.2918 & 0.2596 & 0.2454 & 0.2192 & 0.1628 & 0 \\
\hline & $\overline{\sigma_{\theta z}}$ & 0 & -2.0723 & -2.7911 & -3.1239 & -3.3047 & -3.7157 & -3.3047 & -3.1239 & -2.7911 & -2.0723 & 0 \\
\hline
\end{tabular}

\section{Conclusions}

The present work accounts for the transverse shearing effect on the elastic deformation of simply supported isotropic circular cylindrical shells under static loading by an adoption of a cubic functions expansion of the in-plane displacements. The adopted displacement field accounts for a quadratic (parabolic) distribution of the transverse shear through the shell thickness and satisfies the zero surface stress condition on the top and bottom surfaces of the shell. Analytical solutions were obtained on application of the Navier type solution to the equations governing the deformation of the cylindrical shell. Some numerical examples were considered, evaluated and compared. The incorporation of the transverse effects on the shell's deformation by the present solution is found to result in higher values of the displacements in the moderately thick and thick cylindrical shells in comparison to the Kirchhoff - Love classical theory. However, the classical theory is found to under-predict these displacements within the engineering admissible error (5\%) in moderately thick shells with length to mean radius of curvature ratios within $2.5-10$. The values of the inplane normal and shearing stresses for moderately thick shell predicted by the classical theory is in good agreement to those predicted by the present solution for shells with length to radius of curvature ratios within $2.5-10$. It is concluded that the equations obtained herein could be employed in the static flexural analysis of the circular cylindrical shell of thin to moderate thickness to a sufficient extent.

\section{Nomenclature}

\footnotetext{
$a$ - radius of the circular cylindrical shell

$A, B$ - Lame's parameters
} 


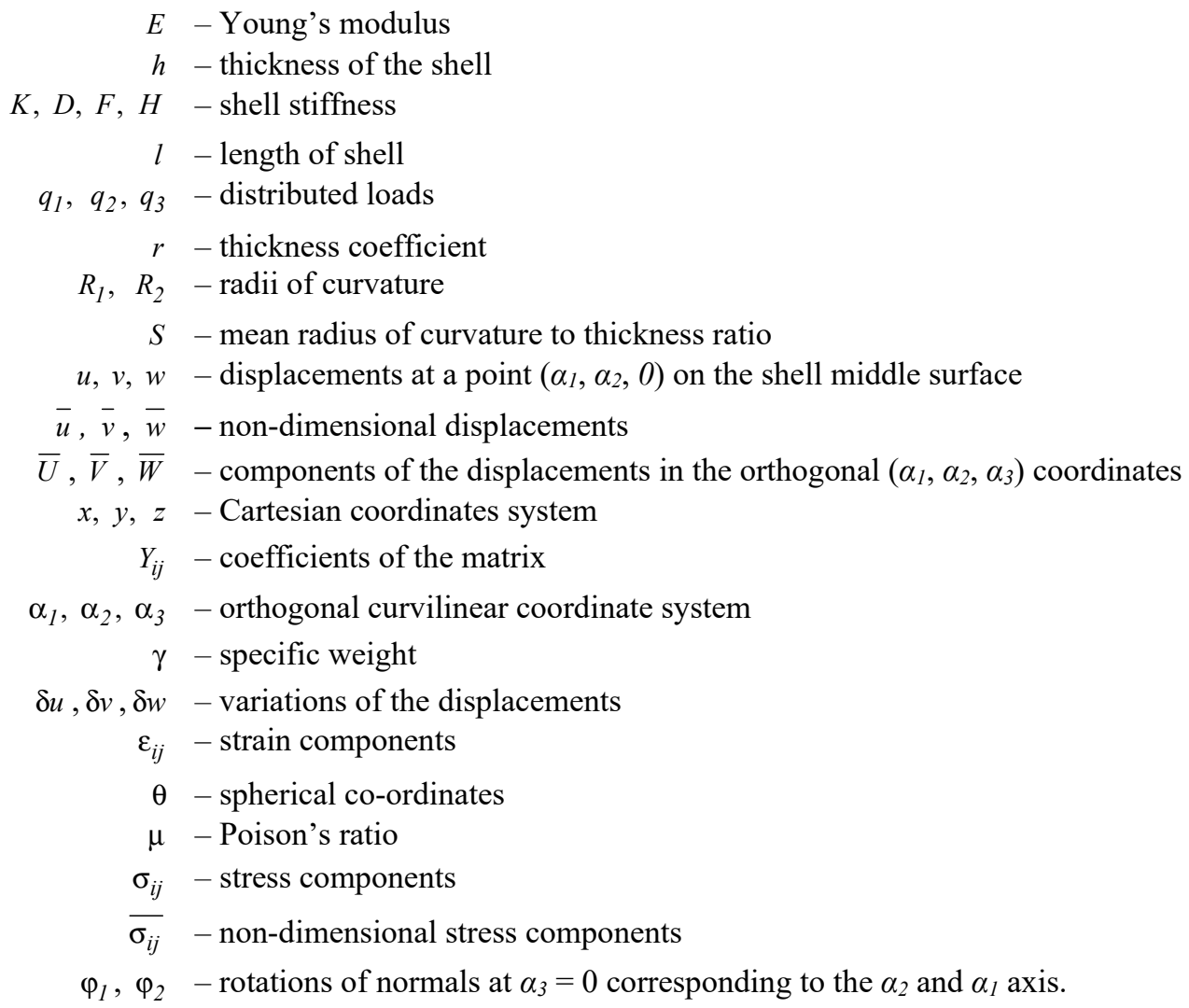

\section{References}

[1] Novozhilov V.V. (1959): The Theory of Thin Elastic Shells.- Groningen: P. Noordhoff Ltd.

[2] Mindlin R.D. (1951): Influence of rotary inertia and shear on flexural motion of isotropic, elastic plates.- Journal of Applied Mechanics, vol.18, pp.31-38.

[3] Selim B.A., Zhang L.W. and Liew K.M. (2016): Vibration analysis of CNT reinforced functionally graded composite plates in a thermal environment based on Reddy's higher-order shear deformation theory.- Composite Structures, vol.156, pp.276-290.

[4] Amabili M. and Reddy J.N. (2020): The nonlinear, third-order thickness and shear deformation theory for statics and dynamics of laminated composite shells.- Composite Structures, vol.244, pp.112265.

[5] Reddy J.N. (1984): Exact Solutions of Moderately Thick Laminated Shells. -Journal of Engineering Mechanics, vol.110, No.5, pp.794-809.

[6] Soldatos K.P. (1986): On thickness shear deformation theories for the dynamic analysis of non-circular cylindrical shells.- International Journal of Solids Structures, vol.22, No.6, pp.625-641.

[7] Reddy J.N. and Liu C.F. (1985): A higher-order shear deformation theory of laminated elastic shells.- International Journal of Engineering Science, vol.23, No.3, pp.319-330.

[8] Di S. and Rothert H. (1995): A Solution of laminated cylindrical shells using an unconstrained third-order theory.Computers and Structures, vol.69, No.3, pp.291-303.

[9] Cho M., Kim K.O. and Kim M.H. (1996): Efficient higher-order shell theory for laminated composites.- Composite Structures, vol.34, No.2, pp.197-212.

[10] Vuong P.M. and Duc N.D. (2020): Nonlinear buckling and post-buckling behavior of shear deformable sandwich toroidal shell segments with functionally graded core subjected to axial compression and thermal loads.- Aerospace Science and Technology, vol.106, pp.106084.

[11] Ventsel E. and Krauthammer T. (2001): Thin Plates and Shells:Theory, Analysis and Applications.- New York: Marcel Dekker. 
[12] Viola E., Tornabene F. and Fantuzzi N. (2013): General higher-order shear deformation theories for the free vibration analysis of completely doubly-curved laminated shells and panels.-Composite Structures, vol.95, pp.639-666.

[13] Soedel W. (2004): Vibrations of Shells and Plates.- New York: Marcel Dekker, Inc.

[14] Ibeabuchi V.T., Ibearugbulem O.M., Ezeah C and Ugwu O.O. (2020): Elastic buckling analysis of uniaxially compressed CCCC stiffened isotropic plates.- International Journal of Applied Mechanics and Engineering, vol.25, No.4, pp.84-95.

[15] Ibearugbulem O.M, Ibeabuchi V.T. and Njoku K.O. (2014): Buckling analysis of SSSS stiffened rectangular isotropic plates using work principle approach. - International Journal of Innovative Research \& Development, vol.3, No.11, pp.169-176.

[16] Ugural, A.C. (2010): Stresses in Beams, Plates, and Shells, Third Edit.- New York: CRC Press - Taylor \& Francis Group.

[17] Amabili M. and Reddy J.N. (2010): A new non-linear higher-order shear deformation theory for large-amplitude vibrations of laminated doubly curved shells.- International Journal Non Linear Mechanics, vol.45, No.4, pp.409-418.

Received: January 16, 2021

Revised: April 15, 2021 